\title{
Deep into the Water: Exploring the Hydro-Electromagnetic and Quantum-Electrodynamic Properties of Interfacial Water in Living Systems
}

\author{
Claudio Messori \\ Str. Villaggio Prinzera 1, Fraz. Boschi di Bardone, Terenzo, Italy \\ Email: messori.claudio@gmail.com
}

How to cite this paper: Messori, C. (2019) Deep into the Water: Exploring the Hydro-Electromagnetic and QuantumElectrodynamic Properties of Interfacial Water in Living Systems. Open Access Library Journal, 6: e5435.

https://doi.org/10.4236/oalib.1105435

Received: April 30, 2019

Accepted: May 28, 2019

Published: May 31, 2019

Copyright $\odot 2019$ by author(s) and Open Access Library Inc.

This work is licensed under the Creative Commons Attribution International License (CC BY 4.0).

http://creativecommons.org/licenses/by/4.0/

\section{(c) (i) Open Access}

\begin{abstract}
Normal water structures are maintained largely by interactions with biomacromolecular surfaces and weak electromagnetic fields, which enable extended networks for electron and proton conductivity. All standard chemistry is totally reliant on electrostatics and avoids all mention of electrodynamics and the consequent radiation field, which is supporting the notion of water as a primary mediator of biological effects induced via electromagnetic means into living systems. Quantum Electrodynamic (QED) field theory have produced a vision of water in a liquid state as a medium, which for a peculiarity of its molecular electronic spectrum reveals itself as an essential tool for long-range communications, being able to change its supra-molecular organization in function of the interaction with the environment. This paper draws attention to the fact that interfacial water (nanoscale confined water) has been shown, independently by Emilio Del Giudice et al. and by Gerald Pollack et al., to contain respectively Coherence Domains (CDs) and Exclusion Zones (EZs), which may be regarded as long-range ensembles of CDs, dynamic aqueous structures, which uses the special properties of water, such as its electron/proton dynamics and organized response to electromagnetic fields, to receive electromagnetically encoded signals endowed with coherence (negentropy) at a low frequency, and sum the resultant excitations, so as to foster the redistribution of that coherence at frequencies which may affect biological systems. The phase transition of water from the ordinary coherence of its liquid state (bulk water) to the semi-crystalline or glassy and super-coherent
\end{abstract}


state of interfacial water and its role in living organisms is discussed. The link between interfacial and intracellular water of the living and 1) the thermodynamic correlation between electron and proton transfer responsible for the redox potential of chemical species, 2) the Grotthuss mechanism and the $\mathrm{H}^{+}$ eightfold path, 3) superconductivity and superfluidity (dissipationless quantum states), 4) the proton motive force and protons role in biological liquid-flow systems, and 5) two possible explanations to as many non-ordinary phenomena, one related to the mind-body severely stressful condition due to a Near Death State (NDS) or to a Near Death Like State (NDLS), namely the Electromagnetic Hyper Sensitivity (EHS) or Electromagnetic After-Effect (EAE), the other related to the harmful consequences avoided during the so-called fire walking (ceremony), are discussed.

\section{Subject Areas}

Chemical Engineering \& Technology

\section{Keywords}

Interfacial Water, Hydrophilic vs Hydrophobic Surface,

Coherence Domain, Exclusion Zone, Proton Transfer, Proton

Motive Force, Grotthuss Mechanism

\section{Introduction}

Water $\left(\mathrm{H}_{2} \mathrm{O}\right)$ is the third most common molecule in the Universe (following the $\mathrm{H}_{2}$ and $\mathrm{CO}$ molecule), and its standard chemical structure, based on the hydrogen bond, is actually confined by a simple scheme of charges interacting via static Coulomb forces; that is, it is totally reliant on electrostatics and omits all mention of electrodynamics and the consequent radiation field. It has been speculated that a goodish percentage of effects in condensed matter physics make use of the radiation field in one way or another but it still doesn't seem to have found a place in much of basic chemistry. In biological systems almost all water is within a fraction of a micron or less from a surface or molecular backbone and so is interfacial water, which behaves in a quantum way, where the Coulomb law of electrostatics does not apply. In these circumstances, like charges attract. Biology itself depends on this, so as to allow the accumulation of tissues from negatively charged cell bodies.

Section 1 of the present work provides an overview of water as seen from both points of view, the one provided by the standard chemistry paradigm and the one introduced by the QED approach.

Section 2 and 3 respectively discuss the meaning of Coherence Domain (CD) introduced by QED and that of Exclusion Zone (EZ) introduced by Gerald Pollack and colleagues.

Section 4 focuses on the thermodynamic correlation between electron and 
proton transfer responsible for the redox potential (oxidation state or electronic configuration) of chemical species. In particular, the Grotthuss mechanism and the $\mathrm{H}^{+}$eightfold path are discussed.

Section 5 provides an overview of two dissipationless quantum states, namely superconductivity and superfluidity.

In Section 6 the proton motive force and protons role in biological liquid-flow systems are discussed.

In Section 7 two possible explanations are given to as many non-ordinary phenomena, one related to the mind-body severely stressful condition due to a Near Death State (NDS) or a Near Death Like State (NDLS), namely the Electromagnetic Hyper Sensitivity (EHS) or Electromagnetic After-Effect (EAE), the other related to the harmful consequences avoided during the so-called fire walking (ceremony), that is how is it possible to walk across a bed of about 482 degrees Celsius (900 degrees Fahrenheit) up to $980^{\circ} \mathrm{C}\left(1800^{\circ} \mathrm{F}\right)$ burning wood coals and emerge unscathed.

Our journey into the water ends up in the deep-sea hydrothermal vent ecosystems, habitats where the unicellular and multicellular organisms inhabiting there can cope with extreme conditions thanks to unusual physiological solutions and food strategies, the understanding of which cannot proceed without the contribution provided by QED.

Emphasizing the central role of water, which in humans constitutes approximately $70 \%$ of total body mass and $99 \%$ of all molecules, marks a departure from the typical molecular biological enzyme-substrate, protein-receptor, and genetic, Watson-Crick base pairing, "lock and key" approach to understanding human physiology and pathology [1] [2].

It is the aim of this paper to introduce an overview on the subject and its relevance in medicine.

\section{Deep into the Water}

Water is a polar molecule, it has positive and negative charges separated by a dipole length and thus exists as an electric dipole. This is due to the $104.5^{\circ}$ angle of the hydrogen bonds to the oxygen atom. The electronegativity of the oxygen atom attracts the electron of the hydrogen atom. Thus the region about the oxygen is negative compared to the region around the hydrogen atoms, which are comparatively positive. Because of this molecular configuration, water molecules mutually attract one another due to the $(-)$ and $(+)$ regions. The negative (oxygen atom) side of a dipolar water molecule attracts and is attracted by any positive ion in solution (ion-dipole force), which, in turn, are attracted to negative ions. This process, in which either a positive or a negative ion attracts water molecules to its immediate vicinity, is called $h y$ dration.

The compound or group that donates the hydrogen is the hydrogen donor, while the compound or group that accepts the hydrogen is the hydrogen accep- 
tor. Water is both hydrogen donor and acceptor; it can donate two hydrogens and its oxygen can accept two other hydrogens. The water molecule is generally represented as a tetrahedron with four "arms"-two hydrogen donors and two hydrogen acceptors-pointing at the vertices. This tetrahedral structure is typical of ordinary ice, where all the water molecules are cross-linked in a crystalline, hexagonal array. The water hexamer, representing a transition from cyclic structures favored by smaller water clusters to 3D structures favored by larger water cluster, is predicted by theory to be the smallest water cluster with a three-dimensional hydrogen-bonding network as its minimum energy structure [3].

Individual water molecules are linked by these hydrogen bonds and form what are called clusters (structural water), tens of nanometers to millimetres in dimensions that can be seen under the transmission electron microscope (TEM) [4] [5] [6].

Water is paramagnetic meaning that it holds a magnetic charge. Para-magnetism occurs primarily in substances in which some or all of the individual atoms, ions, or molecules possess a permanent magnetic dipole moment. Water has a dipole moment and is, therefore, subject to paramagnetism.

Chemical bonds (including covalent, ionic, hydrogen and van der Waals types) have been commonly assumed to be dominating for biological organization and activity. However, these bonds represent forces acting at short distances in the $\mathrm{nm}$ region. Biological systems maintain coherence at every dimension scale. Long-range coherence, large distance cooperation, and the whole body control are significant properties of biological systems.

Water at an interface, as with the atmosphere, has a surface tension due to the polar interactions of water with other water molecules at the interface surface. This clustering imparts a crystalline like property to the water. In the bodies of living organisms, the clusters form hydration layers around biological molecules. It is known from electronics that different patterns which contain information ${ }^{1}$ result within a cluster depending upon its structure. Thus, depending on its structure, each molecule has an oscillatory pattern (resonance frequency) that can be determined by spectroscopy. It is known, through spectrographic analysis, that water and other dipole molecules are able to be entrained to exogenous oscillatory patterns by rearranging their cluster patterns. The cluster rearrangements then resonate with the entraining frequency.

'It must be stressed out that the physical concept of "information" it has absolutely nothing to do with that of "data transmission", and even less with that of "transmission of messages containing a (semantic) meaning". The former (physical concept) consider "information" as a measure of coherence or structural "complexity" of surrounding system related to various entropic processes in physical world, that is the measure of information amount, related to a certain object, may be a complexity of its internal structure (negentropy), while the latter (IT concept) consider "amount ot information" as frequency characteristic of code letters-signals, that is improving of messages coding and decoding methods and solving of other questions related to optimization of technical communication systems operation. 
Like other dipoles, water molecules can stack together in dipole interactions with alternating positive and negative poles next to each other. It can also engage in electrostatic interactions with charged ions and other dipoles dissolved in it. More generally: It is primarily the charge distribution on the surface of molecules that determines their physical and chemical properties, such as bonding ability, orientation, and mutual position. When the geometrical configuration of two molecules fits in such a way that a minimum of the (electrical) Coulomb potentials of the valence electrons is achieved, a chemical bonding can take place. However, charge distribution through its time variations is also responsible for the properties of the e.m. [electromagnetic] radiation which molecules emit, such as polarization, spatial distribution, and direction, and for their interaction with impinging radiation. The interaction of radiation with matter is only possible through redistribution of charge. Structural changes in the molecules entail charge shifts and thus changes in the e.m. field envelope of the molecule, which may be the real mediator of the molecule's interaction with other molecules [7].

Biological systems have high water content containing ions. Ions in water are not just simple charged particles as one would expect to observe in a vacuum, as the charges attract molecules of water that may be bound to them in a variety of configurations and with bonds of varying strength [8].

Hydrogen bonds are non-covalent forces that arise between an acid and a base and may be an intermediary in acid base reactions. Hydrogen bonds provide no net free energy in protein folding but are responsible for aligning atoms and holding them at precise distances and constrain the angle between them. Of particular interest to us are hydrogen bonds to atoms like oxygen, nitrogen, carbon, and sulfur. These bonds are formed when the potential energy wells for a proton in a donor atom overlaps that of an acceptor atom so that the barrier between them is low enough to allow the transfer of protons. The forces of attraction are largely electrostatic in nature and vary with distance as the interaction between dipoles is shielded by the dielectric constant of the medium.

Carignano et al. [9] investigated the effect of the ionic polarizability on the solvation of positive and negative ions in water, and he concludes that increases of the polarizability lead to a larger electrical field at the ion. This occurs through shrinking of the solvation shell around the ion and the asymmetric location of the ion in the cage. Positive ions have smaller polarizabilities than negative ions. However, for a given polarizability, the electrical field at an ion and probability of asymmetric location is larger for cations than for anions.

Ion-specific effects are widespread, but nowhere are more critically manifested than at the fluid interfaces of biological structures. Action potentials, osmotic flows, energy transduction, and the stabilization of proteins are driven by ion concentration gradients across liquid films on hydrophobic biomaterials. Recent experiments [10] revealed that ions interact specifically at the prototype air-water interface over separations that vastly exceed the range of direct elec- 
trostatic forces in any dielectric medium ${ }^{2}$. Such long-range specific ion effects may be triggered by electrostatic and electrodynamic forces, but they must be powered also by other mechanisms, such as the thermal fluctuations intrinsic to fluid interfaces.

As a liquid, water possesses many remarkable properties that to a large extent can be explained by its extremely high density of hydrogen bonds. One of these properties is the anomalously high mobility of protons and hydroxyl ions $\left(\mathrm{OH}^{-}\right)$ in this liquid. This phenomenon has been explained from a (Grotthuss) conduction mechanism that involves the exchange of the chemical O-H bond and the hydrogen bond in the $\mathrm{O}-\mathrm{H}$-..O hydrogen-bonded system formed by an $\mathrm{H}_{3} \mathrm{O}^{+} / \mathrm{OH}^{-}$ion and an $\mathrm{H}_{2} \mathrm{O}$ molecule. Metabolic/energetic regulation may well depend on the flow of protons (proton currents) and on other nonlinear optical and phonon effects such as solitons via liquid semi-crystalline water (which is sharing all the characteristics of soft matter, as for colloids, polymers, gels and foams $s^{3}$ ) [15], structured in nanospaces throughout the extracellular matrix into the interior of every single cell and its nanospaces [16]. Moreover, the separation of positive and negative charges is important for short and long range collective coherent correlation (SLRCCC), especially in the form of proton-SLRCCC.

Relatively little is known about the effects of hydrogen bond interactions in liquid water on the reactivity of the O-H groups of the water molecule [17] [18] [19], and despite intense theoretical and experimental study, it continues to hold some surprises, e.g. hydrogen bonded protons in liquid water experience significant excursions in the direction of the acceptor oxygen atoms, generating a small but non-negligible fraction of transient autoprotolysis events, associated with major rearrangements of the electronic density [20].

In most molecular dynamics simulations, these effects are not included. Even

${ }^{2}$ Dielectrophoretic attraction of dielectric particles to living cells is observed, and a corresponding frequency of oscillations is assessed in the frequency range 1.5 - 52 MHz. Recently, researchers have directed their efforts to distinguish between healthy and pathological state of cells utilizing a technique called dielectrophoresis (DEP), an analytical diagnostic and screening technique that uses the principles of polarization and the motion of bioparticles in applied electric fields [11], that is by detection of cells' dielectric and electrophysical properties (dielectrophoretic profile) [12] [13], managing to clarify the processes underlying cell membrane impairment due to electrical and mechanical stress (electro-deformation) [14]. DEP is the movement of particles by a trapping force in a non-uniform electric field when the particles and surrounding medium have different polarizabilities. According to the Maxwell-Wagner theory of conductivity in heterogeneous systems, there is a critical frequency that separates the low-frequency range, where ionic conductivity dominates, from the high-frequency range, where dielectric properties determine the system's behavior in the electric field.

${ }^{3}$ All the major constituents of living organisms, from lipids of cellular membranes to DNA, possibly all proteins, especially cytoskeletal proteins, muscle proteins, and proteins in the connective tissues such as collagens and proteoglycans, may be liquid crystalline. Liquid crystals (LCs) are states or phases of matter in between solid crystals and liquids, hence the term, mesophases. Unlike liquids which have little or no molecular order, LCs have orientational order, and varying degrees of translational order. But unlike solid crystals, LCs are flexible, malleable, and responsive. LCs typically undergo rapid changes in orientation or phase transitions when exposed to electric (and magnetic) fields. They also respond to changes in temperature, hydration, shear forces and pressure. Biological LCs carry static electric charges and are therefore also influenced by $\mathrm{pH}$, salt concentration and dielectric constant of the solvent. 
in the most advanced Car-Parrinello molecular dynamics simulations [21] these effects are not well accounted for because the nuclear coordinates are described classically. In view of the small mass of the hydrogen atom and the proton, such a classic approach gives a poor description of the properties of the $\mathrm{O}-\mathrm{H}$ groups of the water molecule. The effects of hydrogen bonding on the $\mathrm{O}-\mathrm{H}$ bonds of water can in principle be studied by a spectroscopic investigation of the different vibrational quantum states of the $\mathrm{O}-\mathrm{H}$ stretch vibrations. Unfortunately, such a study is strongly complicated by the ultrafast (subpicosecond) energy equilibration of liquid water [22]. Therefore, the experimental study of the excited vibrational states of the hydrogen-bonded $\mathrm{O}-\mathrm{H}$ groups of the water molecule requires the use of ultrafast (i.e., femtosecond) mid-infrared (mid-IR) spectroscopy. This type of spectroscopy has been applied successfully to the study of the vibrational relaxation [23] [24] [25], molecular reorientation [26], and hydrogen bond dynamics [27] [28] of different isotopic varieties of liquid water.

In biological systems, liquid water interacts not only with small solutes but also with many larger, extended hydrophilic and hydrophobic surfaces, such as those of proteins, nucleic acids, various organelles, and cell membranes [1].

Results of inelastic incoherent neutron scattering studies of several cell and tissue types suggest that ca. $20 \%$ - 30\% of the total (intracellular plus extracellular) water in these systems is interfacial water, i.e. water located within $1-4 \mathrm{~nm}$ of these surfaces, with bulk water comprising the remaining $70 \%-80 \%$. At this nanoscale level, interfacial water near hydrophilic surfaces displays viscosity from about 2 up to $10^{6}$ times greater than that of bulk water, while no significant water viscosity changes are seen near hydrophobic surfaces [29]. Furthermore, water near extended (>ca. $1 \mathrm{~nm}$ ) hydrophobic surfaces shows less hydrogen bonding and behaves more like water near a liquid-vapor interface than bulk water [30].

However, biological water can be considered as (structured)interfacial water, due to the fact that there is almost no point in an organism that is not far more than a fraction of a micron from a surface.

According to [1] the main systems by which (structured) interfacial water promotes life-enabling biological processes include:

- promoting electrical conductivity at biological interfaces, thereby facilitating metabolism and voltage differences maintained by intracellular organelles;

- absorbing, storing, and emitting electromagnetic energy, enabling storage and transmission of energy and information;

- overcoming the kT or "thermal diffusion" problem; and

- solving the intracellular crowding and molecular self-assembly problems by way of chirality (handedness of molecules) [31] and magnetization.

Exogenous interfacial water stress (EIWS), may disrupt biological water structure, initiating a series of events in extracellular and intracellular space leading toward disorder and disease, such as neuropathologies, infections, cancers, and fatalities [1] [32] [33]. 
Disruptive changes may occur in the aqueous interphase ("interface" refers to the surface area between two phases, while "interphase" corresponds to the volume defined by the narrow region sandwiched between the two phases ${ }^{4}$ ), the zone near a biomacromolecular surface where water structure and properties differ from those of bulk liquid water. Disruptive changes consist of:

- Life-enabling water structures in the aqueous interphase, normally maintained by weak magnetic fields and the heparan sulfate proteoglycans (HSPGs) that decorate cell membrane surfaces, are disrupted by exogenous interfacial water stressors such as aluminum cations.

- This disruption leads to localized water hydrophobicity, unwetting, increased water tension, and membrane "softening." In addition, exogenous interfacial water stressors such as aluminum cations ties up cell surface HSPGs by charge neutralization and thus breaks up the HSPG-membrane complex that connects extracellular matrix components to the intracellular cytoskeleton.

- The resulting disconnection of the cytoskeleton from the plasma membrane has several adverse consequences, including impaired electrical conductivity of the cytoskeleton and microtubules ${ }^{5}$ and re-orientation of the cytoskeleton toward the cell nucleus, which can accelerate the pathological mitosis characteristic of cancer.

${ }^{4}$ The concept of a soft interface is related to the notion of "interfaces and interphases" [34]. This homophone pair appears to originate from adhesion science, and has been applied in different context. In this notion, the transition zone of finite width between two phases is addressed as a separate entity having its own character. This "interphase" is not a true phase in the thermodynamic sense, but it is not a purely two dimensional separation sheet between the two neighbouring bulk phases either. The layer between the bulk phases can be affected by external fields. Here we look for concepts to increase its response. Interface tension plays the role of the major antagonist against interface fluctuations. It aims to keep the interface area as small as possible. Interface bound fluctuations of large thermal amplitude must not significantly affect the interface area. Consequently, only interfacial degrees of freedom which are weakly coupled to the interface area can become soft, except for low interface tension systems. The inverse interface tension corresponds to the low compressibility in bulk systems. In bulk, only fluctuations which do not involve compression acquire large thermal amplitudes.

${ }^{5}$ Energy is supplied to microtubules by hydrolysis of GTP upon $\beta$-tubulin polymerization, through motion of motor proteins, and by nonlinear transfer from the higher frequency oscillations. This is where many diseases (which has been shown to be a result of mitochondrial disruption) connection comes in. When mitochondrial function slows, the infrared light and electronegative outputs of the cell drop and so does the volume of structured water (EZ) [35]. Much of the cytosolic water is created as a byproduct of mitochondrial respiration and electron chain transport. This metabolism makes heat (bio-photons emission and phonons transfer) and the density of electrons flowing along the inner mitochondrial membrane correspond the electronegative charge (which makes proteins hydrophilic). Mitochondria transport protons into the intermembrane space, and their diffusion into cytosol leads to generation of a strong static electric field and water ordering. The strong static electric field shifts also vibrations in microtubules into a highly nonlinear region. The decrease of oxidative metabolism results in changes of the intensity of the static electric field and changes of water ordering. The water ordering depends also on $\mathrm{pH}$ factor and on the static electric field.

In presence of dysfunctional mitochondria, the surrounding biological water's layer reorganize with a reversed orientation of the electric field, which enables transport of electrons released into cytosol. As biological water occupies $70 \%$ of the cell volume, it is capable of releasing a huge amount of electrons into the cytosol. Free electrons increase conductivity which causes damping of electromagnetic field. The mechanism of damping electromagnetic oscillations generated by microtubules may explain the disturbed organization in cells with dysfunctional mitochondria [36] [37]. 
- In addition, penetration of the interfacial water stressors into the cell disrupts intracellular water structure, leading to unfolded protein response, unfolded DNA response, and excess reactive oxygen species (ROS) production.

Interfacial water assumes a glassy appearance, that is semi-crystalline, and has been studied by several researchers [38], suggesting the possibility that it is of a different phase (Pollack's four phase) from that of common water in the liquid state [39].

Neither classical nor standard quantum theory predicts quantum coherence for water, largely because they ignore quantum fluctuations and the interaction between matter and the vacuum electromagnetic field(VEMF), which are taken into account in Quantum Field Theory (QFT).

\section{Water Coherence Domains}

QFT explicitly recognizes an extended VEMF interacting with matter, as well as quantum fluctuations whereby energy in the VEMF in the form of photons could be captured by matter. The first clear example was the Lamb shift, the energy of an electron surrounding the proton in a hydrogen atom is slightly lower than the value calculated from the atomic theory based on purely static forces. Although this shift is very small, it provided evidence of the quantum vacuum fluctuation that has to be understood within the framework of Quantum Electrodynamic (QED) field theory. In the case of the hydrogen atom, the effect is due to the interactions between the electric current of the electron orbiting the nucleus and the fluctuating EMF of the surrounding space (vacuum). For a collection of particles, the usual approach is to apply the Lamb shift to each particle separately. While this is correct for very low density systems like gases, where the distance between any two particles is larger than the wavelength of the relevant fluctuating fields coupled to the systems, dense systems-condensed matter or liquids and solids-show entirely different behavior. When energy is absorbed from the VEMF, the particles will begin to oscillate between two configurations. In particular, all particles coupled to the same wave-length of the fluctuations will oscillate in phase with the EMF, that is, they will be coherent with the EMF. At that point, a phase transition occurs. The coherent oscillations of the particles no longer require any external supply of energy, they become stabilized and will begin to attract more molecules and attract each other, thereby turning gas into liquid in a change of phase. With further increase in density, the system becomes a net exporter of energy because the stabilized coherent state has a lower energy than the incoherent ground state. A collection of molecules interacting with the radiative EMF above a density threshold and below a critical temperature acquires a new minimum energy state different from the conventional where the oscillations of individual molecules are uncorrelated and the electromagnetic field is vanishing. The new minimum energy state is a Coherence Domain $(\mathrm{CD})$ that oscillates in unison and in tune with an EMF trapped within it. 
The physical process can be therefore summarized as follows. The system has initially the configuration where all particles are independent and the EMF is absent; subsequently it runs away from this configuration, driven by the exploding EMF and finally settles in the configuration described by the limit cycle. This limit cycle describes just a coherent situation since the fields, both EM and matter, have defined phases and moreover the frequency of the EMF happens to be equal to the difference of the frequencies of $\chi_{0}$ and $\chi_{q}$, which is the frequency of oscillation of the molecular system. Therefore molecules and EMF oscillate in tune. Finally it is possible to show that energy per particle of the system, after inserting the limit cycle fields, assumes a negative value, which means that the phase transition is a spontaneous process. The EMF is trapped in the $\mathrm{CD}$ because in the limit cycle its frequency decreases sharply producing on the internal border a situation of total reflection. The field therefore falls off exponentially out of the $\mathrm{CD}$, giving rise to an evanescent field. This field acts as a trap for surrounding molecules so that the molecule density in the coherent state increases until reaching a saturation value when the intermolecular distance reaches the value of the radius of the molecular hard core [40].

In the QFT approach, the quanta of the field correlating the molecules are components of the system on the same ground that the molecules, and they would disappear when the system is dismantled. In QFT the interaction is considered an object as much as the basic components. Moreover, the tight binding between molecules and the correlation field produces new basic objects named quasi-particles and the conventional separation between matter and interaction is dropped out. An important feature of the QFT approach is the fundamental role assumed by the physical variable, named the phase $\Phi$ of the field (which should not be confused with the thermodynamic phase). The phase $\Phi$ describes the rhythm of oscillation of the field and therefore the wavelike aspects of the system.

Contrary to the objects described by Classical Physics, a coherent quantum system is not defined in isolation, but gets defined by the array of its relationships. The phase $\Phi$ is connected with the EM potential in a mutual relationship so that we could be able to change the phase of a biological organism by applying an EM potential [41] [42] [43].

We could interpret the biological effectiveness of very and ultra weak EM and magnetic fields just by assuming that the agent at work in the interaction is not the field but the potential and the mechanism of interaction is the phase-sharing ${ }^{6}$.

${ }^{6}$ External electromagnetic signals can be selectively damped by tissues, according to their being or not in phase with the possible oscillatory motion of the system's components. This specific phase-matching (i.e. resonance) feature operates as a very selective mechanism, a sort of filter discriminating among perturbations and stimuli acting on the system, thus protecting it against any noisy perturbative background or even strong actions, which, however, are out of phase with the oscillatory motions allowed by the system's inner dynamics [44]. 
That is, the biological organisms, being coherent, can interact with environment in two basically different ways:

- through the conventional exchanges of energy which amount to the application of mutual forces. This mechanism of interaction obeys to the causality principle, since energy cannot travel faster than light (c);

- through the sharing of the phase $\Phi$ with other coherent systems (biological organisms), which amounts to the establishment of a resonance with them [45].

The phase velocity is not bounded above and can be larger than $c$.

In the framework of a biology based on coherence, the health of an organism depends on its ability of constructing a well defined phase during the mainly unpredictable variables of life. When this happens, we say that this organism is able to self adapt and, eventually, self repair. The capability of self repair of an organism is enhanced by the presence of a well defined phase in tune with the part of the environment the organism is connected to. However, in case of severe diseases, the chemical structure of the organism could become so modified to be unable to resonate with its background water; in these cases of course a chemical repair seems to be necessary.

A better life quality is then supported by a sharper definition of the phase of each organism. In this interaction the connectedness of the organism is essential, not the amount of exchanged energy, which could on the contrary be harmful when its amount exceeds the threshold of the "energy gap", namely the amount of energy necessary to make the coherent system boiling and therefore losing coherence [40].

In standard QFT, the energy levels of material systems are shifted by their interaction with the fluctuations of the electromagnetic field(EMF) in the vacuum. QFT predicts that liquids, being condensed matter with high density, are not governed by purely static local interactions such as $\mathrm{H}$-bonds and dipoles. On the contrary, their binding is induced by radiative long range electromagnetic fields (LR-EMF). But the conventional QFT applies only to gases.

The conventional $a b$ initio approaches to water, based just on Quantum Mechanics (QM), describe it as a monophasic liquid. On the contrary, the conceptual frame of QFT admits infinitely many ground states (vacua), each one corresponding to a particular function describing the expectation value of the involved field.

Quantum fluctuations and couplet between matter and VEMF in QED indeed predicts quantum coherence for liquid water even under ordinary temperatures and pressures. QED has introduced the concept according to which the interaction between the vacuum EMF and liquid water induces the formation of large, stable Coherence Domains (CDs) of about $100 \mathrm{~nm}$ in diameter at ambient conditions, and these CDs may be responsible for all the special properties of water including life itself.

According to Giuliano Preparata, Emilio Del Giudice and colleagues [2], the 
water $\mathrm{CD}$ is a quantum superposition of ground coherent state and excited state (in the proportion of 0.87 and 0.13 ). Liquid water is therefore a two-fluid system consisting of a coherent phase (about 40 percent of total volume at room temperature) and an incoherent phase. In the coherent phase, the water molecules oscillate between two electronic configurations in phase with a resonating EMF. The EMFs that are trapped within the CD of water and within its coherent matrice [46], produce electromagnetic potentials that regulate the phase of the entire system, which in turn gives rise to selective attractions between the molecules of the solute. The CD is then a resonating cavity produced by the EMF (a self-produced cavity resonator for the EMF), whose size is just the wavelength $\lambda$ of the trapped EMF, that ends up trapping the field because the photon acquires an imaginary mass, so the frequency of the CD electromagnetic field ${ }^{7}$ becomes much smaller than the frequency of the free field with the same wavelength. Within the CD water molecules oscillate between the ground state and an excited state close to the ionizing potential of water and, therefore, contain close to a million almost-free electrons. That means CD is most likely negatively charged at the periphery close to or at the surface of its domain (at the same time, positively-charged protons are present just outside the CD).

Thus pure bulk liquid water consists in two interspersed phases, coherent and incoherent, having widely different dielectric constants ${ }^{8}$ (that of the coherent

\footnotetext{
${ }^{7}$ Electromagnetic field generated in living cells and conditioning biological activity is a nature of life. In eukaryotic cells, the electromagnetic field is generated by microtubules composed of tubulin heterodimers with a strong electric dipole. Energy transport, processing, parceling out into small bits and storing into adenosine triphosphate (ATP) and guanosine triphosphate (GTP) form a complex fermentative and oxidative pathway. High-energy electrons are transported down the respiratory chain in the mitochondrial inner membrane, and the released energy is used to pump protons across the inner membrane into the intermembrane space and cytosol. The electrochemical gradient around mitochondria is formed. In functional mitochondria, the actual electric inner membrane potential of the electrochemical gradient is about $-140 \mathrm{mV}$ and the $\mathrm{pH}$ gradient of about $-1 \mathrm{pH}$ unit. Resonant frequencies of microtubules are important parameters for assessment of the cellular functions and interactions with other cells in the tissue. Interaction between cells is mediated by cellular electromagnetic field in the near-infrared range. Microtubules are capable to generate electromagnetic field in a wide spectrum in classical frequency bands up to $20 \mathrm{GHz}$, at $20 \mathrm{THz}$, and in the UV range. Comparing to healthy cells, unhealthy cells aren't getting enough electrons and radiant energy (e.g. in the form of cells and tissues' biophotons emission and ELF-UV light from cell's nucleic acids, or in the form of solar light), lowering their volume of EZ water.

${ }^{8}$ One way of thinking about the dielectric constant (despite a massive amount of literature dedicated to the subject, the dielectric constant of interfacial water and its depth remain essentially unknown because measurements are challenging [47]) is to think of it as the fraction of the electric field that is shorted out by the movement of charged particles that are limited in the extent of their motion. In the case of the water structures mentioned earlier (water hexamer), the dielectric constant can be thought of as resulting from the movement of hydrogen ions from one end to the other or the induction of a dipole moment across the structure. This structure with an induced dipole moment may also rotate to align along the field. The average size of these structures can be expected to decrease as the temperature increases as the thermal energy available to break hydrogen bonds increases. The fraction of the dielectric constant contributed by the ability of these structures to short out the electric field would be expected to decrease as the temperature increases. The different sized structures can be expected to have different time constants for both the motion of the hydrogen ions and the rotation of the structure.
} 
phase is 160 , due to the high polarizability of the coherently aligned water molecules that are oscillating in concert; while the dielectric constant of the incoherent state is about 15). The incoherent phase comprises water molecules in the molecular ground state (as observed in the gas phase) packed in a highly dense state in the interstices around large clusters in which the water molecules perform hindered rotations and interact coherently with a large electromagnetic field.

The externally applied electric fields are therefore only felt in the non-coherent phase. Because coherent water is excited water with a plasma of almost free electrons, it can easily transfer electrons to molecules on its surface. The interface between fully coherent interfacial water and normal bulk water becomes a "redox pile".

All biological envelopes, from cell membrane to epithelial tissue, contain this aqueous phase in a liquid-crystalline state or are perfused by it. It is water in a particular phase of quantum organization (oscillatory coherence) that close to charged hydrophilic surfaces is confined in layers of Exclusion Zone (EZ) (see next paragraph), and gives it a high capacity to:

- retain electronic charges, in the form of vortical excitations of quasi-free electrons, storable as energy reserve;

- induce an electronic and protonic long-range and long life excitation of the different molecular species available, enabling their selective activation and mutual attraction;

- convert mechanical vibrations (phonons) in quanta of electromagnetic energy (photons) ${ }^{9}$ and viceversa (piezoelectric effect).

The CD of liquid water, unlike the $\mathrm{CD}$ of other molecular species, is susceptible to give rise to a large number of excited states [48]. Arises, consequently, the possibility of a further level of coherence, generated by the collective oscillation of a plurality of CDs of water, between two own configurations: a coherence between CDs, namely Super-coherence [49], that on one hand makes it grow the size of the related region by the tenth of a micron of the elementary CDs of water, up to microns of the cells, to centimeters of the organs, or to the meters of higher organisms.

Given the plurality of the excited levels of the CD, it is able to withdraw from the environmental noise small amounts of energy, transforming them into coherent vortices of quasi-free electrons. The duration of these vortical excitations can be very long (days, weeks, months), since because of the coherence the internal friction is zero, there are no collisions and the CD can not dissipate energy in thermal form. Given the long duration of these excitations, it is possible to accumulate a large number of them within the domain. Each vortex is a motion of electrons, that is, electrically charged particles, which gives rise to the appear-

${ }^{9}$ Coherent mechanical vibrations (phonons) of living cells are measured by atomic force microscopy in the acoustic frequency range. Frequencies of the mechanical vibrations and of the electromagnetic field (photons) generated by a cell are equal. 
ance of a magnetic moment, which in turn aligns with the environmental magnetic field, which in the final analysis can also be the Earth's magnetic field ${ }^{10}$ [57] [58]. The vortices therefore can not cancel each one another, but add up coherently, then transforming low-quality environmental energy (high entropy), in high quality coherent energy (low entropy-neghentropy), capable of inducing, as predicted by Szent-Gyorgyi [59], the electronic excitation of the molecules surrounding the CD/EZ. There it exists at normal body temperature, with its density fluctuations between a dominant coherent low entropy and a less frequent incoherent distorted high entropy ordering water. The former is organized in spherical CDs of clustering water molecules tuning in unison their quantum oscillations in phase with a self-trapped EMF within the CDs, forming an inclusive endo-plasma. The CDs can be activated to collect in their environment low-grade energy with high entropy, and transform it, by exciting coherent vortices of almost free electrons, into high-grade energy, with low entropy, so that this energy can be released outwards into useful work without thermal losses [60]. Energy of thermal fluctuations is then transformed into energy of the super-coherent state [43] [48] [61].

The unique value of such super-coherent biological water spanning all the CDs throughout water can be considered one of the most important characteristics of a healthy physiological state.

\section{Interfacial Water Exclusion Zones}

In the frame of QED, then, the dominant contribution of water molecules in

\footnotetext{
${ }^{10}$ In order to load energy in the water CDs, a resonating magnetic field is needed. In higher organisms, such as humans, these fields can be induced by the nervous system. In elementary organisms, such as bacteria or yeast cells and physiological liquids, i.e. water, environmental fields like geomagnetic fields can serve as the inducer [50]. These modes act as stationary fields produced by the magnetic activity occurring in the shell whose boundaries are the surface of the earth-conductive ionosphere resonant cavity, which acts as a mirror wall for very-low-frequency (VLF) and Alfvén waves [51]. Aqueous solutions of bicarbonates, superoxide radicals, and other ROS, when excited, show variations in energy-emitting activity that have been found to correlate with fluctuations in the geomagnetic field [52]. Accordingly, bacterial and viral DNA sequences have been found to induce low-frequency EMFs in high aqueous dilutions [53]. The formation of condensed DNA copies was triggered by the ambient geomagnetic EMF background of VLFs related to the Schumann resonances, specifically the $7.8 \mathrm{~Hz}$ band. In humans, physiological rhythms and global collective behaviors are not only synchronized with solar and geomagnetic activity, but disruptions in these fields can also invoke adverse effects upon human health and behavior [54]. It is well established that the resonant frequencies of geomagnetic Ultra Low Frequencies (ULF) $(0.006-0.2 \mathrm{~Hz}$ ) overlap closely with the frequencies of the cardiovascular system $(0.002 \mathrm{~Hz}-0.2 \mathrm{~Hz})$, while Schumann resonances $(7.8-51 \mathrm{~Hz}$ ) directly overlap with those waves of the human brain (Theta $4-7 \mathrm{~Hz}$, Alpha $8-12 \mathrm{~Hz}$, Beta $12-30 \mathrm{~Hz}$, and Gamma $30-100 \mathrm{~Hz}$ ). As stated above, to get a collective performance of water $\mathrm{CDs}$, which can give rise to resonance with intrinsic VLF rhythms, would require a uniform rate of energy loading from, e.g., a magnetic field for all involved CDs. A plausible interaction with geomagnetic ULF can affect the human cardiovascular system, because several ULFs are in a comparable range with those of the human heartbeat and its rhythms [55]. The external non-local background load of conditioned EMF photons in water CDs is in resonance with, e.g., the frequency of ULF geomagnetic field pulsations that can be biotropic [56], specifically, stable continuous pulsations.
} 
molar terms and total mass in living systems makes water the apparent element that can give rise to a non-linear self-regulative quantum super-coherent state, and to a nest of CDs of water [31], whose properties overlap to some extent those of interfacial water EZs, or $4^{\text {th }}$ phase of water, proposed by Pollack and colleagues [62], by inducing coherence among CDs forming a systemic axial coherence, such as the biological water state of the human body.

At charged surfaces, water super-coherent layers are called Exclusion Zones (EZs may be regarded as long-range ensembles of CDs), as solutes are excluded from them [63] [64].

EZ interfacial water is electrically charged with respect to bulk water, which is well known to be neutral. If EZ interfacial water, in vitro, is close to a surface bearing a net negative charge, it acquires a negative charge, too, whereas it acquires a positive charge near a surface bearing a positive charge. It is interesting to observe that when EZ interfacial water becomes negatively charged near a negative surface, positive charges (protons) appear beyond EZ interfacial water on the side of the EZ layer facing bulk water. On the contrary, negative charges appear on the side of the layer facing the bulk water when EZ interfacial water is positively charged. It is also interesting to realize that no electric opposite charges have been detected in the interstice between the solid-like surface and the aqueous surface, suggesting that the attraction water-solid surface underlying the hydrophily of the surface is not produced by electrostatics but by a dynamic collective attraction such as the one produced by QED coherence [65].

The properties of EZs have been widely investigated by the group led by G.H. Pollack [66] [67] [68]. By using dyes dissolved in water as a probe, his team was able to detect the existence of extended regions in the boundary between the liquid and the wall of the container, where the dyes were prevented from entering (EZs), provided that the wall was an hydrophilic surface. The depth of EZs could reach a length of some hundreds of microns (with macroscopic thicknesses up to about $500 \mu \mathrm{m}$ ), much longer than the estimates of conventional studies on liquid water. For instance, in the computational scheme presented by Buch et al. [69] the interfacial layers are defined to contain 60 molecules, whose total size cannot exceed a couple of hundreds of $\AA$, a length smaller than the observed depth of the EZ layer by four orders of magnitude.

However, the established physical properties of EZ interfacial water are summarized as follows [63] [70]:

- EZ interfacial water is considerably more viscous than bulk water (about 10-fold) [65].

- EZ interfacial water has a negative electric potential (up to $200 \mathrm{mV}$ ) with respect to the neighboring normal bulk water; thus the pair EZ interfacial water-bulk water is a redox pile. This property of EZ interfacial water could account for the source of electron excitations.

- Protons concentrate at the boundary between EZ interfacial water and bulk water.

- EZ interfacial water exhibits a peak of light absorption at $270 \mathrm{~nm}$; it emits 
fluorescence when excited by light having this wavelength.

- The illumination of EZ interfacial water by light (especially IR radiation) increases the depth of the layer.

- EZ interfacial water cannot host solutes.

The above list of properties appears quite mysterious in the frame of conventional ideas about liquid water (monophasic liquid), while is well explained in the frame of QED (biphasic liquid).

Biological EZ interfacial water is always negatively charged at the periphery close to or at the surface of its boundary, and the amorphous enclosed molecules are always positively charged. Interfacial water is ubiquitous in all biological environments and involved in all major cellular processes, implying that it might be a common target for the known effects (e.g. the killing of bacteria, changing the growth rate of plants and insects, various physiological and behavioral changes in animals including humans) of atmospheric electricity (positive and negative atmospheric ions). Generally speaking, negative atmospheric charge is believed to promote good health, whereas positive charge compromises health [71]-[76]. Nature provides a reasonably balanced supply of air ions; however more negative ions are found at places such as waterfalls and forests [77], which often elicit sensations of well-being. The question arises whether these effects are largely psychogenic or whether some physical mechanism exists to explain the effects. However, there is still no agreement to date on the mechanism of how the charged air ions affect biological system. According to recent studies [78] beyond a threshold concentration the ill effect of atmospheric positive charge on interfacial water, which is found to have a net negative charge, may become appreciable-even wiping out the negativity of interfacial water and replacing it with the many positive ions in the water (this phenomenon can potentially explain the "refreshing" sensation that people often feel when stepping out of a crowded room with positive ions from exhalation, and/or with positive ions produced from an air conditioner, and into fresh air. Once the inhibiting positive ions are removed, the interfacial water structure can easily and quickly rebuild. In terms of health impact, this suggests that restoration of function can occur rapidly once the positive air ions are removed. When the conditions are right, enough air ions could be produced to change the electrical properties of the interfacial water.

\section{The Grotthuss Mechanism and the $\mathrm{H}^{+}$Eightfold Path}

Both biomolecules and isolated water molecules are not electron donors, since electrons are tightly bound to parent molecules with binding energies of several eVs. In the conventional theory of liquid water, this paradox cannot easily appear, since the existence of the liquid is taken for granted (no description is provided for the dynamics of the phase transition vapor-liquid and the consequent large increase in density) [70].

Starting from an ensemble of molecules, which are already close enough to 
stay within the range of static interaction, the computer simulation calculates the shape of the network formed by a small number of molecules (at most one thousand). Hence, the probability of the movement of protons along the network is estimated through computer simulation; in this way it has been found that it is possible to recover the mechanism introduced by Grotthuss 200 years ago. In the conventional approach the importance of the collective effects has been recognized. The difference between the conventional approach and the QFT approach is just in the size of the aggregates of molecules. The aggregates emerging from the $a b$ initio calculations, which use static interaction, only have a size of a few tens of $\AA$ at the most, whereas the water CDs, according to QED, span over $0.1 \mu \mathrm{m}$ and include millions of molecules. The conventional approach introduces the a priori not unreasonable approximation that only the static part of the interaction is relevant. The QFT approach includes also the non static interaction, which has a much longer range than the static one, namely the EMF, which is the field that modern quantum physics considers responsible for the interaction between particles, in this case the molecules are not at rest but are subjected to quantum and thermal fluctuations.

Furthermore, water electricity is special in that it also involves, as we have seen early, the movement of positive charges associated with protons. According to QED not only do electrons of the hydrogen bonds fail to conform to the classical electrostatic model, the protons also are quantum mechanical. Changes in the redox potential or proton transfer equilibria of a chemical species can influence each other, thereby these two chemical reactions often occur in association. This fundamental concept of chemistry is best exemplified by the Nernst Equation which relates the variation of the aqueous oxidation/reduction potential of chemical reactions with the $\mathrm{pH}$ when protons are involved. It is known that cells can exist and perform particular functions in complex environments within a particular range of temperature and $\mathrm{pH}$ conditions [79]. The $\mathrm{pH}$ level is different for different parts of the body. Thus for example, in order to decompose food to basic components, the stomach maintains an acidic environment. The cells, covering inner walls of stomach, must be resistant to these extreme conditions and the proteins and receptor-ligand complexes within should be able to perform their functions. Another example are immunocompetent cells, such as monocytes and neutrophils. While being activated, they produce reactive oxygen species that acidify the environment. Thus, the change in $\mathrm{pH}$ is used as a powerful weapon against pathogen organisms. In addition, these cells perform phagocytosis, at which monocytes and neutrophils capture the pathogens inside phagosomes inside cells. Furthermore, the $\mathrm{pH}$ value is not constant throughout the cell, compartments of the cells can have widely differing $\mathrm{pH}$.

In a more general sense, the thermodynamic correlation between electron and proton transfer establishes that the redox potential (oxidation state or electronic configuration) of a chemical species can affect its acid-base equilibria, that is, its $\mathrm{pK}(\mathrm{a})$ (or protonation state) [80]. This concept constitutes the thermodynamic 
basis of proton-coupled electron transfer (PCET) reactions [81] [82], also called Concerted proton electron transfer (CPET), in which proton and electron movement are intercorrelated.

Most widespread chemical substance in the living body is liquid water, which consists primarily of a mixture of clusters of water molecules with different degrees of hydrogen bonding in an equilibrium. Under thermal fluctuations some hydrogen couplings are broken but other arise. On average, the equilibrium distribution of different cluster sizes is maintained. In this fluid medium, the hydronium ion, $\mathrm{H}_{3} \mathrm{O}^{+}$, is a carrier of protons, that is hydrogen ions $\mathrm{H}^{+}$. Proton exceeds the electron mass on about 2000 times. It means that proton is a more inertial particle than electron, and, consequently, more robust for thermal fluctuations. For that reason, hydrogen ion can be adopted as the unit of thermal motion [83]. The hydrogen bond is strong enough to maintain the coupling of atoms during some time under thermal fluctuations. The strength of the H-bond has important implications for proton transfer (PT) kinetics since it correlates with the proton free energy profile and the PT energy barrier. Very weak $\mathrm{H}$-bonds are typically associated with asymmetric (different donor and acceptor moieties) single-well proton free energy profiles and high PT barriers. Progressive increase of the H-bond strength changes the proton free energy profiles towards asymmetric double-well, to symmetric double-wells with a concomitant decrease of the PT barrier. The unusual case of very strong H-bonds, features symmetric single-well proton free energy profiles. In this particular case the energy minimum corresponds to the proton centered in the middle of the H-bond and therefore no PT transfer barrier exits [84] [Figure 1; Figure 1(a)].

Thanks to the hydrogen-bonded chain mechanism [85] [86], called the Grotthuss mechanism, protons tunnel from one water molecule to the next via hydrogen-bonding [87]. Surprisingly, excess protons can create their own pathways, water-wires, before protons can migrate along [88].

According to the Grotthuss mechanism in the context of transition to the superconductivity, once a hydrogen ion has passed in one direction, the other ion cannot pass. However, the latter can go along the same water-wire in the opposite direction, meaning that the Grotthuss mechanism switches the water-wire in backward and forward directions after each was passed by the $\mathrm{H}^{+}$. It turns out that there can be such an organization of water, when the Grotthuss mechanism is able to support a long-living hydrogen ion current. This organization is due to the hexagonal circuits characterizing the coherent (ordered) organization of water EZ [89], the hexagonal skin or layer of hydrogen and oxygen molecules which surround an amorphous arrangement of water molecules.

The EZ network of hexagonal layers is so densely packed (liquid crystalline layers), and the symmetry is so uniform, that no other particles than hydrogen and oxygen are allowed to penetrate. These properties provide favorable conditions for the Grotthuss mechanism. The negative charge of EZ indicates the existence of many holes-empty seats with a negative charge where the hydrogen 


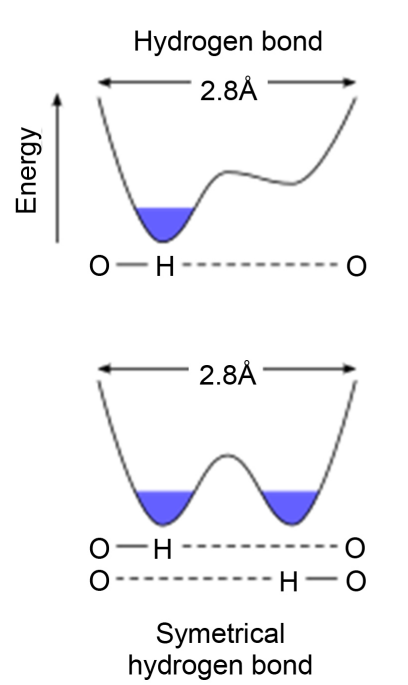

Low-barrier

hydrogen bond
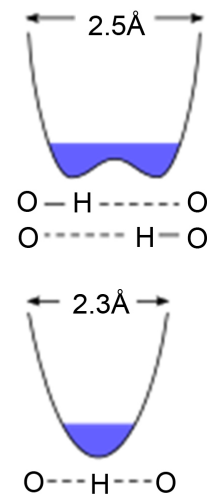

Single-well hydrogen bond

(a)

The importance of strong $\mathrm{H}$-bonds in chemical kinetics The strength of the $\mathrm{H}$-hond is an important factor also in chemical kinetics because the $\mathrm{H}$-hond strength, the shape of the PT profile, and the height of the PT barrier are strietly related, PT barriers being large in weak aSW $H$-bonds, rapidly decreasing in strong DW $\mathrm{H}$-bonds, and finally disappearing in very strong and proton-centred sSw $\mathrm{H}$-bonds.

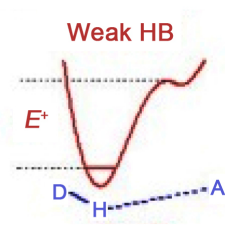

asW-HB

metric Single-Well Asymmetric Double-

High-Barrier

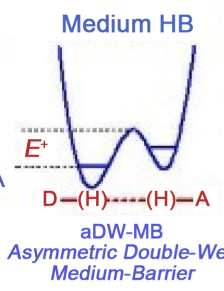

As a consequence,

the H-bond enthalpy $\Delta H_{\square}$ basically determines the eight of the PT barrier ( $\left.E^{*}\right)$ and this, in turn, the PT rate constant $\left(k_{P T}\right)$ through the Arrhenius equation.

This fact is of outstanding importance in chemical kinetics because proton transfer is an important step in many chemical and biochemical reactions and, in particular, in general acid-base catalysis where protonation of the substrate is mostly assured by ancillary $\mathrm{H}$-bond formation.

(b)

Figure 1. (a) Low-Barrier Hydrogen Bonds (L-BHBs). Image source:

https://en.wikipedia.org/wiki/Low-barrier_hydrogen_bond. (b) The importance of strong H-bonds in chemical kinetics. Credit: Gastone Gilli University of Ferrara Department of Chemistry and Centre for Structural Diffractometry. Image source:

https://slideplayer.com/slide/7249780/.

ion may hop. Hence we can consider the Grotthuss mechanism as counter-movements of the hydrogen ions and their holes.

Long-lived current can exist due to realization of the eightfold path of the $\mathrm{H}^{+}$ in the paired hexagonal circuits [Figure 2]. In this organization all hexagonal circuits form a giant hexagonal packed circuit, where the currents circulate around the hexagonal circuits by the Grotthuss mechanism and by the torque generation mechanism [90] [Figure 3], showing some meaningful analogies with the spiraling eightfold path of the Twisted-Pinched Hysteresis Loop [91] 
(a)

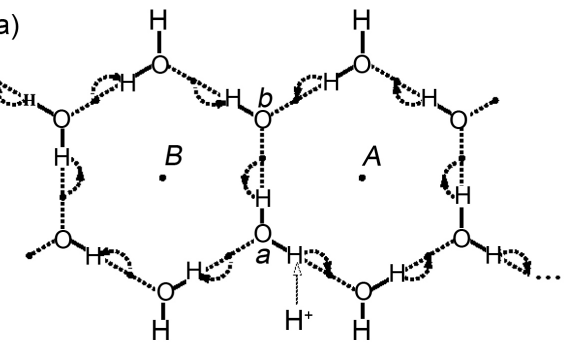

(b)

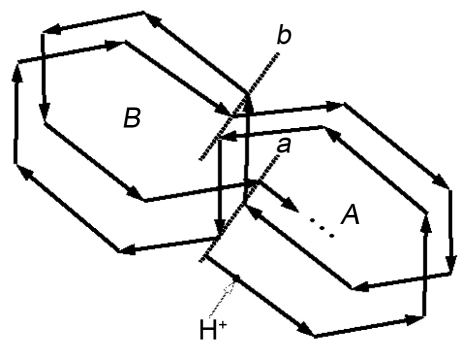

Figure 2. The eightfold path. (a) According to the Grotthuss mechanism, by hopping a hydrogen ion bypasses the hexagonal circuits around A and B and changes the direction of the bypass on the bifurcation nodes a and b; (b) a conditional diagram showing the journey of the hydrogen ion along the hexagonal circuits marked by A and B. The path bifurcates on the nodes $\mathrm{a}$ and $\mathrm{b}$ each time as soon as the hydrogen ion reaches them (the dotted lines conventionally depict also a shift in time). Credit [83]. Image source: https://www.semanticscholar.org/paper/Quantum-consciousness-in-warm\%2C-wet\%2Cand-noisy-brain-Sbitnev/0a71d78bd4c5f30bb895fb1216366e1bfa32c06f.

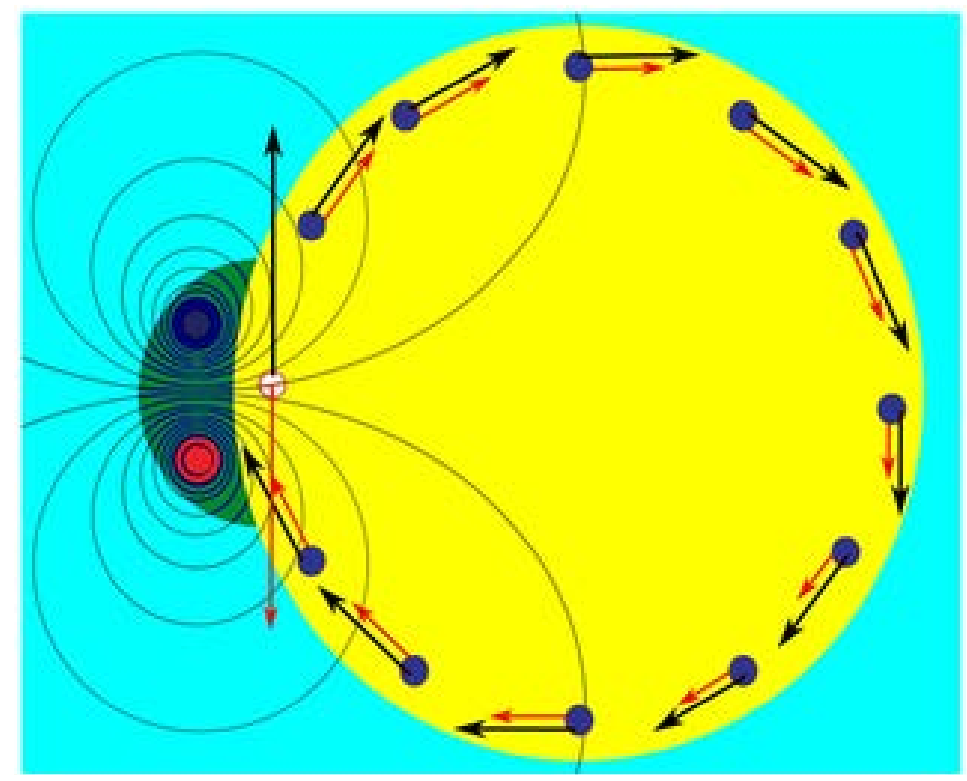

Figure 3. During ATP synthesis, the ion motive force across a mitochondrial, bacterial, or thylakoid membrane drives the $c$-ring of FO ATP synthase to rotate. Top view of the $c$-ring (yellow) and stator a-subunit (green) of FO, showing equipotential surface cross-sections (curved lines) perpendicular to the electric field emanating from the half-channels (blue and red circles) in the a-subunit. Black arrows represent forces due to tangential field components (red arrows) acting on protonated (blue circles) and deprotonated (light circle) sites on the $c$-ring. The figure shows cross-sections of the resulting equipotential surfaces (black lines) and tangential electric field components (red arrows), superimposed on an idealized cross section of the c-ring (yellow) and a-subunit (green). The proton channel cross sections in the a-subunit are colored to depict the differences in potentials, with dark blue and red representing the channels coupled to high- and low-potential sides of the membrane, respectively. The protonated sites on the $c$-ring are shown as dark blue circles, while the light circle represents a deprotonated site. The black arrows represent tangential forces due to the field acting on protonated and deprotonated sites. Crucially, both can make positive contributions to the torque since opposite field directions are counterbalanced by opposite charges. Credit [90]. Image source: https://journals.plos.org/plosone/article?id=10.1371/journal.pone.0074978. 
[Figure 4] and with the vortex-antivortex pairs of superfluid vacuum [92] [93] [94].

The $\mathrm{H}^{+}$travels on the water-wire passing through two hexagonal circuits, in such a way that an open path for the ion always exists.

A model of proton-conducting water chain or proton-wire has come from a further unexpected source: studies on carbon nanotubes [95] [96] [97].

The fast exchange of protons in the interfacial water (remember all water in living organisms can be considered as interfacial water) may also be an indication of a non-classical proton jump-conduction extending throughout the interfacial water. Water "jump" conducts protons down a chain of water molecules connected by hydrogen bonds, in which a proton leaps on at one end of the chain, and a second leaps off at the other end, while electrons are displaced in the other direction. A form of semi-conduction much faster than electrochemical conduction through nerves, because the charge displacements involved are very short ranged.

Nevertheless, there are indications that water could play essential role in superconductivity (see next paragraph for general insights on the subject). As a coherent quantum phenomenon, superconductivity could be responsible for coherent behavior of living organisms.

In 1964, William A. Little proposed the possibility of high and very high-temperature superconductivity in organic polymers [98].

This proposal is based on the exciton-mediated electron pairing, as opposed to phonon-mediated pairing in Bardeen, Cooper and Shrieer theory (BCS-theory).

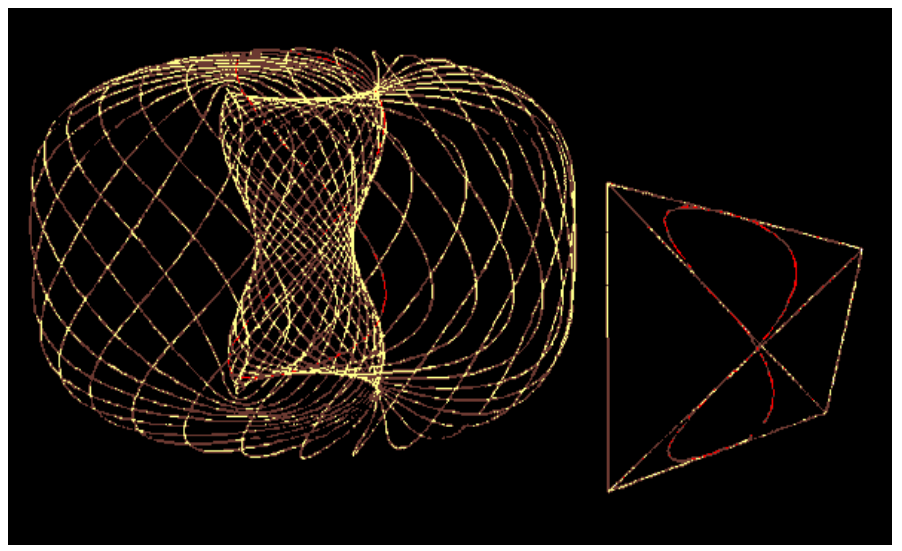

Figure 4. Biaxial or tetra-toroid, also coined as external toroid warped around an internal poloid, as drawn here has 27 identical loops. Compared with ordinary toroid coil, the main differences are twisted loops instead of the plain toroid loops and the involuted "donut hole". While 27 closed loops are presented to show a tetrahedron relationship, all loops can be one continuous twisting line. Itsstructure corresponds to aholographic cavity resonator with toroidal-poloidal topology consisting of an ongoing Twisted-Pinched Hysteresis Loop (T-PHL) [91] hysteresis cycle wrapped around an energy-free wormhole (void) consisting of tension gradient distribution. Image source: http://harmoniouspalette.com/TetMold.html. 
He proposed that an arrangement of one-dimensional organic chains in a polarizable medium was a promising candidate for high-temperature superconductivity. Little predicted that the large polarization could be provided by side chains surrounding the conducting chains, which would induce (via polarization excitations) a great effective attraction between electrons in the conducting chains, leading to pairing (a necessary condition for superconductivity) at high temperatures.

Although several organic superconductors have been synthesized, Little's prediction has been, from a conventional point of view (namely in the frame of molecular biology), undermined by the effects of electron instabilities in the structural and transport properties of low-dimensional systems [99], while in the frame of QED this instability is nullified by the EZs/CDs interfacial water coherent dynamic [100].

However, what makes organic superconductivity distinctive is that conduction in organic molecular conductors, which reveal quasi-one-dimensional features (examples for low dimensional physics) of their electronic transport properties due to their peculiar "crystal structure", is linked to the transport of free charges (electrons or holes) between $\pi$-like molecular orbitals of neighbouring open shell molecules.

In the very tightly confined space inside microtubules and interface's EZs, water is structured in a specific way by entangled $\mathrm{H}-\mathrm{O}$ chains. Such chains could have dangling electron-proton bonds linked to well-ordered. This could provide strong electron-electron and proton-electron interaction necessary for superconductivity with very high critical temperature (Tc), namely the temperature at which a phase transition occurs and the electrical resistivity of a non-superconductor drops to zero turning it in a superconductor, like it is in the model suggested by W.A. Little. Alternative explanation is that in the confined space of microtubules and interface's EZs, each atom of oxygen is surrounded by several atoms of hydrogen. It was already demonstrated that in hydrates, Tc is dramatically increasing with hydrogen coordination number [101].

The confinement into nano-channels and nano-layers may lead to extremely high critical temperature (room-like Tc). The properties of individual microtubules with and without water were measured in [102] [103].

It was shown that water is responsible for high conductivity of microtubules, and their coherent electrical behavior was also demonstrated.

\section{Superconductivity and Superfluidity: General Insights}

Now it is clear that resistance of a superconductor should be exactly equal to zero. This follows the fact that the current flow through the superconductor is based on a quantum effect. The behavior of electrons in a superconductor are therefore governed by the same laws as in an atom.

Therefore, in this sense, the circulating current over a superconductor ring is analogous to the movement of electrons over their atomic orbits.

Boris V. Vasiliev [104]. 
Since its discovery in 1911, superconductivity was considered to be essentially low-temperature phenomenon. The critical temperature (Tc) of the first superconducting material, $\mathrm{Hg}$, was about $4 \mathrm{~K}$. In the atom, the passage of an electron from one orbit to another corresponds to a phase transition. The passage of a material from the state of normal conductor to superconductor is associated with a change (i.e. phase transition) order of its electronic subsystem, that is, transition occurs as an ordering in the electron subsystem. Accordingly, superconductivity is due to the fact that its carriers have charge 2e-, i.e., they represent two paired electrons. As a consequence, the pairing of electrons is a necessary condition for the existence of superconductivity [104] [105].

Superconductivity is a phenomenon of exactly zero electrical resistance and expulsion of magnetic flux fields occurring in certain materials, called superconductors, when cooled below a characteristic Tc and/or when subjected to a critical pressure (from high to extreme). A superconductor is generally considered "high-temperature" if it reaches a superconducting state when cooled using liquid nitrogen — that is, at only $\mathrm{T}>77 \mathrm{~K}$-or "low-temperature" if more aggressive cooling techniques are required to reach its critical temperature. A room-temperature superconductor is a material that is capable of exhibiting superconductivity at operating temperatures above $0^{\circ} \mathrm{C}(273.15 \mathrm{~K})$. While this is not strictly "room temperature", which would be approximately $20^{\circ} \mathrm{C}-25^{\circ} \mathrm{C}$ $(293.15-298.15 \mathrm{~K})$, it is the temperature at which ice forms and can be reached and easily maintained in an everyday environment [106].

Like ferromagnetism and atomic spectral lines, superconductivity is a quantum mechanical phenomenon (superconductivity and superfluidity are both dissipationless quantum states). The electrical resistance of a metallic conductor decreases gradually as temperature is lowered. In a superconductor, the resistance drops abruptly to zero when the material is cooled below its Tc. An electric current through a loop of superconducting wire can persist indefinitely with no power source. All superconductors have exactly zero resistivity to low applied currents when there is no magnetic field present or if the applied field does not exceed a critical value. The onset of superconductivity is accompanied by abrupt changes in various physical properties, which is the hallmark of a phase transition. For example, the electronic heat capacity is proportional to the temperature in the normal (non-superconducting) regime. At the superconducting transition, it suffers a discontinuous jump and thereafter ceases to be linear. At low temperatures, it varies instead as $\mathrm{e}-\alpha / \mathrm{T}$ for some constant, $\alpha$. This exponential behavior is one of the pieces of evidence for the existence of the "energy gap". The existence of these "universal" properties implies that superconductivity is a thermodynamic phase (a new state of matter), and thus possesses certain distinguishing properties which are largely independent of microscopic details.

A major step forward in the theoretical understanding of superconductivity was made in 1940 by L. Landau. Landau explained the superfluidity of helium-II, 
discovered two years earlier by Pyotr L. Kapitsa, treating helium-II as a substance where the laws of quantum physics worked on a macroscopic scale. This phenomenon is akin to superconductivity: the superconductivity can be regarded as the superfluidity ${ }^{11}$ of an electron liquid. As a result, the relationship between phenomena have much in common, with some differences, since both phenomena are described by the same quantum mechanics laws in macroscopic manifestations.

As Boris V. Vasiliev points out [105]:

Superfluidity and superconductivity, which can be regarded as the superfluidity of the electron gas, are related phenomena. The main feature of these phenomena can be seen in a fact that a special condensate in superconductors as well as in superfluid helium is formed from particles interconnected by attraction. This mutual attraction does not allow a scattering of individual particles on defects and walls, if the energy of this scattering is less than the energy of attraction. Due to the lack of scattering, the condensate acquires ability to move without friction. (...) However, despite the attention of many scientists to the study of these phenomena, they have been the great mysteries in condensed matter physics for a long time. (...) The mystery of the superconductivity phenomenon has begun to drop in the middle of the last century when the effect of magnetic flux quantization in superconducting cylinders was discovered and investigated. (...) By these measurements it became clear that at the formation of the superconducting state, two free electrons are combined into a single boson with zero spin and zero momentum.

H. Fröhlich (1950) was the first to point out that at low temperatures, the interaction with phonons can lead to nascency of forces of attraction between the electrons, in spite of the Coulomb repulsion.

A few years later, L. Cooper predicted the specific mechanism in which an arbitrarily weak attraction between electrons with the Fermi energy would lead to the emergence of a bound state. On this basis, in 1956, Bardeen, Cooper and Shrieer built a microscopic theory (the so called BCS-theory), based on the electron-phonon interaction as the cause of the attractive forces between electrons.

According to the BCS-theory:

- The attraction in the electron system arises due to the electron-phonon interaction. As result of this attraction, the ground state of the electron system is

\footnotetext{
${ }^{11} \mathrm{~A}$ superfluid is a state of matter in which the matter behaves like a fluid with zero viscosity.Such a fluid flows without loss of kinetic energy and without friction past any surface, being able to continue to circulate over obstructions and through pores in containers which hold it, subject only to its own inertia. When stirred, a superfluid forms cellular vortices that continue to rotate indefinitely. Superfluidity occurs in two isotopes of helium (helium-3 and helium-4) when they are liquified, by cooling to cryogenic temperatures. In the frame of Quantum Field Theory helium-3 and helium-4 in the liquid state are considered a two-phase liquid (which require the existence of a mesoscopic/macroscopic correlation field). It is also a property of various other exotic states of matter theorized to exist in astrophysics (neutron stars), high-energy physics (Superfluid Vacuum Theory) and theories of quantum gravity. The phenomenon is related to Bose-Einstein condensation, but neither is a specific type of the other: not all Bose-Einstein condensates can be regarded as superfluids, and not all superfluids are Bose-Einstein condensates.
} 
separated from the excited electrons by an energetic gap. The existence of energetic gap explains the behavior of the specific heat of superconductors, optical experiments and so on.

- The depth of penetration (as well as the coherence length) appears to be a natural consequence of the ground state of the BCS-theory.

The most important yet negative role, which plays a major part in the development of the science of superconductivity, is the isotope effect (a measure of the contribution of phonons to the pairing mechanism), which was discovered in 1950. The negative role, of the isotope effect is played not just by the effect itself but its wrong interpretation. This effect has made researchers think that the phenomenon of superconductivity is actually associated with the vibrations of ions in the lattice, but the difference of the isotope effect in different superconductors could not be explained by phonon mechanism.

The coupling of electrons in pairs can be the result not only of electron-phonon mechanism. Any attraction between the electrons can lead to their coupling. For the existence of superconductivity, the bond energy should combine into single ensembles of separate pairs of electrons, which are located at distances of approximately hundreds of interatomic distances. In BCS-theory, there are no forces of attraction between the pairs and, especially, between pairs on these distances.

Until 1986, physicists had believed that BCS-theory, which explained the superconducting current as a superfluid of Cooper pairs (pairs of electrons interacting through the exchange of phonons), forbade superconductivity at temperatures above about $30 \mathrm{~K}$. In that year, Bednorz and Müller discovered superconductivity in a lanthanum-based cuprateperovskite material, which had a transition temperature of $35 \mathrm{~K}$. It was soon found that replacing the lanthanum with yttrium raised the critical temperature to $92 \mathrm{~K}$. Many other cuprate (compounds of copper and oxygen) superconductors have since been discovered, and the theory of superconductivity in these materials is one of the major outstanding challenges of theoretical condensed matter physics.

There are currently two main hypotheses:

- the so called resonating-valence-bond theory [107], and

- the hypothesis proposing that electron pairing in high-temperature superconductors is mediated by short-range spin waves known as paramagnons [108].

At the beginning of the twenty-first century the assumption developed in the middle of the last century, that the electron-phonon interaction is the only possible mechanism of superconductivity was proved to be wrong. At very low temperatures, that allow superfluidity in helium and superconductivity in metals, all movements of particles are "freezed" except for their zero-point oscillations. Therefore, as an alternative, we should consider the interaction of super-particles through electromagnetic fields of zero-point oscillations. Thus it is possible to show that both related super-phenomena, superconductivity and su- 
perfluidity, are based on the single physical mechanism: the ordering of zero-point oscillations. The vacuum zero-point energy fluctuations happen on surface of a vast ocean of energy called by Fedi the superfluid quantum space [109] [110]. It contains virtual particle-antiparticle pairs, which are created and annihilated again and again, possessing integer spin and therefore forming the Bose-Einstein condensate covering the entire Universe. The principal condition for the superconducting state formation, that is the ordering of zero-point oscillations, is realized due to the fact that the paired electrons, which obey Bose-Einstein statistics, interact with each other. As they interact, their amplitudes, frequencies and phases of zero-point oscillations become ordered.

Quoting Vasiliev [105]: J. Bardeen was first who turned his attention toward a possible link between superconductivity and zero-point oscillations. The special role of zero-point vibrations exists due to the fact that at low temperatures all movements of electrons in metals have been frozen except for these oscillations. Superconducting condensate formation requires two mechanisms: first, the electrons must be united in boson pairs, and then the zero point fluctuations must be ordered. The energetically favorable pairing of electrons in the electron gas should occur above the critical temperature. Possibly, the pairing of electrons can occur due to the magnetic dipole-dipole interaction. (...) It is agreed that the superconductivity can occur only in metals with two collectivized electrons per atom, and cannot exist in the monovalent alkali and noble metals.

The most significant discovery within the past 30 years is that water has quantum properties under ambient conditions, and that water confined in nanospace is both quantum coherent [111] and proton semi-conducting by quantum delocalization, that is, a proton can be in two places (delocalized) along the $\mathrm{O}-\mathrm{H} \cdots \mathrm{O}$ axis linking up the covalent bond of the donor water molecule and the hydrogen bond to the acceptor molecule, in other words the excited proton can be found simultaneously at a distance of the equilibrium $\mathrm{O}-\mathrm{H}$ bond from the left oxygen in $\mathrm{O}-\mathrm{H} \cdots \mathrm{O}$, and at the same distance from the right oxygen (in the two potential wells), hence increasing the probability of proton transfer [20] [112], which goes beyond classical jump conduction (diffusion of the ions in a ionic crystal via the points defect). However, a new scenario linked to room-temperature superconductivity and superfluidity at ambient pressure is on the stake, where also protons, and not just electrons, can travel unobstructed at room temperature through crystalline water layers confined in nanospace.

According to QED [113] [114] the behavior of water is analogue to the one of superfluid helium-4: there are two fractions, coherent and incoherent, and they increase or decrease between 0 and $100^{\circ} \mathrm{C}$ as helium- 4 fractions. Furthermore, the boundary-water interface confinement it excite the ground state of water (changes in the electronic bond structure) to coherent quantum delocalization of the proton, making it superconducting protons. A possibility, nowadays, in line with Reiter et al. prediction [16]: There is experimental confirmation of correlated proton motion in the vibrational spectrum of ice. We hypothesize that a 
similar phenomenon is occurring in water, with the correlated motion of the protons producing a response of the electrons that leads to an effective double well for the protons, and that this correlated state, while occurring at higher energies... than the individual molecule state of bulk water, is sufficiently close in energy that it can become the ground state when the hydrogen bond network of bulk water is disrupted by confinement. The changes in the zero-point motion [ground state] of the protons in confined water, as in living cells, for instance, can be expected to play a significant role in the energetics of the cells, where typical distances between components are on the order of $20 \AA$.

\section{The Proton Motive Force and Protons Role in Biological Liquid-Flow Systems}

In most chemical systems, nuclear quantum zero-point motion and tunneling do not play a significant role. Most of chemistry can be understood in terms of semi-classical motion of nuclei on potential energy surfaces. In contrast, the quantum dynamics of protons involved in hydrogen bonds plays an important role in liquid water [20] [115], ice [116] [117], transport of protons and hydroxide ions in water [118], surface melting of ice [119], the bond orientation of water and isotopic fractionation at the liquid-vapour interface [120] [121], isotopic fractionation in water condensation [122], proton transport in water-filled carbon nanotubes [96] [123] [124], hydrogen chloride hydrates [125], proton sponges [126] [127], water-hydroxyl overlayers on metal surfaces [128], and in some proton transfer reactions in enzymes [129].

Proton currents and concentration gradients are ubiquitous in all biological systems and play essential roles in a number of physiological processes [130]. The most striking example is oxidative phosphorylation in mitochondria in which proton gradients serve as a means to translate the energy from oxidation of glucose during the Kreb's cycle into ATP, the biological energy currency [131] [132].

According to British biochemist Peter Mitchell's chemiosmotic theory (1961) [133], who won a Nobel Prize for this, living systems uses proton gradients to build potential energy through electrostatic tension. Living systems are charged up predominantly by accumulating protons on one side of a membrane, and discharged by protons flowing back down to the other side. Protons are transported across biological membranes by special membrane proteins called proton pumps. The protons pumped uphill (to a higher energy state), using an external energy source, such as the oxidation of foodstuff, or absorption of sunlight, is returned downhill via another enzyme, ATP synthase embedded in the same membrane, which uses the energy to make ATP, the universal energy intermediate that powers all living activities. The protons are supposed to exist in bulk solution on either side of the membrane, and it is the difference in concentration between the two compartments separated by the membrane that drives the synthesis of ATP. 
Mitchell's chemiosmotic theory put forward the idea that ATP synthesis is driven by a proton electrochemical gradient between two cellular compartments across the biological membrane. This proton electrochemical gradient $\left(\Delta \mu \mathrm{H}^{+}\right)$, which is often expressed as proton motive force ( $\mathrm{pmf})$, is a proton $\left(\mathrm{H}^{+}\right)$motive force generated across biological membranes by electron-transport-linked proton translocation, acting as the intermediate that couples the electron flow through the respiratory chain with ATP synthesis by ATP synthase in the mitochondrial matrix across the inner mitochondrial membrane. ATP synthase is highly relevant to medicine due to its crucial bioenergetic role in humans, and its dysfunction has been implicated in cancer [134] [135], neurodegenerative diseases [136] [137], and other illnesses.

According to Mitchell's proton motive force equation, pmf is a thermodynamic potential that has two components: an electrical component represented by $\Delta \psi$, which is the trans-membrane potential generated due to the difference in electrical potential across the membrane, and a chemical component represented by $\Delta \mathrm{pH}$ which is the difference of protons concentration between the two bulk aqueous phases separated by the membrane [138]. However, Mitchell's pmf equation underestimates the true transmembrane proton motive force which is due to the delocalization view of excess protons that was postulated by Mitchell in his theory. Furthermore, Mitchell's chemiosmotic theory does not clearly explain what really define or contribute to the membrane potential $\Delta \psi$.

Since the chemiosmotic hypothesis was first proposed, it has been suggested (R.J.P. Williams, 1993) that the protons, rather than accumulating in solution in the bulk of the cell compartment, actually diffuse along the membrane surface.

In $2012 \mathrm{~J}$. W. Lee published a new hypothesis named proton-electrostatics localization hypothesis (P-ELH) [139]. This hypothesis provides a possibly unified explanation of the proton localization and/or delocalization in all bioenergetics systems within bacteria, mitochondria and thylakoid structure (the site of the light-dependent reactions of photosynthesis inside chloroplasts and cyanobacteria) in the chloroplast of plants without requiring any proton sub-compartments or interfacial proton barrier. The P-ELH is based on two assumptions. The first assumption is that excess protons in aqueous phase can quickly transfer among water molecules throughout the hydrogen bond network by "hops and turns" mechanism which was first outlined by Grotthuss. The second assumption is that liquid water can be considered as a proton conductor. Since the excess protons mobility in water is very fast, excess protons in liquid water can be treated like the excess electric charges in a metallic conductor where Gauss law can be applied on them. According to Gauss law, for electrostatic charge distribution in a general conductor, excess electric charge in a conductor at equilibrium will reside on the surface of the conductor body and not in the bulk. This is expected because the freely moving excess electrons repel one another and arrange themselves on the surface to suffer the minimum possible repulsion. Similarly, applying Gauss law on excess protonic charge in the aqueous proton conductor will 
lead to electrostatic localization of excess protons on the water surface along the water-lumenal interface of the water membrane.

And that's what they are doing, they distribute themselves to the water membrane interface at the lumenal side of the membrane where they attract electrostatically the excess hydroxyl anions $\left(\mathrm{OH}^{-}\right)$at the stromal side of the membrane, forming an excess protons-membrane-excess anions capacitor-like system. Therefore, a proton capacitor concept can be used to determine the effective localized proton concentrations at the membrane-water interface. According to Lee's equations there is a close relationship between the localized excess proton concentrations and the membrane potential $\Delta \psi$ : it is the localized excess protons, the thickness for localized proton layer and the membrane capacitance that define the membrane potential $\Delta \psi$. Therefore, the proton-electrostatics localization hypothesis can be considered as a significant development over the chemiosmotic theory.

When protons diffuse along the surface of membranes (two-dimensional diffusion) instead of through the bulk solution (three-dimensional diffusion), the rates of proton transport processes are significantly increased [140]. This is due to a fundamental difference of diffusion in two as opposed to three dimensions. In three dimensions, a proton far away from its target-say, at the entrance to a proton pump embedded in the membrane-will have a very small probability to be caught by the target. But in two dimensions, the probability of the proton being caught is exactly 1 . And if instead of random diffusion, protons are jump-conducted along chains of interfacial water molecules aligned along the membrane surfaces (as it happens within the liquid crystalline matrix of biological water), then proton transport processes can indeed be quite fast.

Thus, confinement leads to excitation of water and quantum delocalization of the proton, greatly facilitating proton conduction.

However, proton transfer:

- Is involved in regulating enzyme reactions within the cell, where metabolic reactions are predominantly of a redox nature.

- Proton currents may well flow throughout the extracellular matrix, and linked into the interior of every single cell through proton channels.

- Proton currents could flow from the most local level within the cell to the most global level of the entire organism.

- Protons (reducing power) give a boost of energy where it is needed.

- Protons can flow directly along the membrane within the interfacial water layer, from proton pump to ATP synthase, both of which are embedded in the membranes.

But proton currents and concentration gradientsplay essential role also for itcan mimic a motive-like pump that turns any biological liquid flow collagen-related system in a self-driven liquid-flow system. That is a light-driven-flow [141].

In order to clarify this role, let us remember that EZ is built by hydrophilic 
material and radiant energy spanning from the infrared to the UV range, and that connective-collagen membrane system happens to be composed almost entirely of hydrophilic material. Hydrophilic materials are defined by the density of electrons on their surfaces, and the protons formed when 1) ultraviolet to infrared light energy, 2) water, and 3) hollow hydrophilic surfaces come together in space and time.

Collagen is the most abundant protein in the organism, and is known to form liquid crystalline mesophases (remember that liquid crystals are states or phases of matter in between solid crystals and liquids, hence the term, mesophases). It is the main protein in the extracellular matrix and connective tissues and may thus account for the liquid crystallinity of living organisms as a whole, facilitating SLRCCC (short and long range collective coherent correlation) throughout the body. Type I collagen is the archetype of all collagens as well as the most abundant. It is found in tendons, skin, vasculature as well as the organic portion of the calcified tissue of bone and teeth. What makes collagen most interesting is its associated biological water [142]. Most of the H-bonds in collagen structure are water mediated. Water chains mediate H-bonding between carbonyl groups on the same chain as well as between different chains in the triple helix, and between the $\mathrm{OH}$ group of hydroxyproline with carbonyl groups in the same or different chains. The number of water molecules involved in bridging two groups appears to vary along the helix. Water bridges are also critical in connecting adjacent triple-helices and maintaining the molecular spacing. Local hydrogen-bonding network is observed in the interstitial waters. Some water molecules link up to four other water molecules, illustrating the three-dimensional hydrogen-bonded network of water around the collagens.

The structured water associated with collagen is highly ordered as a single phase at any time, and at critical points of hydration, abrupt phase transition occurs to a different state of order, in analogy with ice structures. Evidence dating back to the 1970s [143] indicates that collagen does conduct protons, while evidence dating back to the late 1980s indicates its capacity for second harmonic generation (SHG), i.e. combining photons interacting with it to form new photons with twice the energy and, therefore, double the frequency and half the wavelength [144] [145].

Now, the highly ordered collagen water chains, which are linked to Pollack's fourth phase of water, are reminiscent of those seen in carbon nanotubes $(<5 \mathrm{~nm}$ diameter).

Under certain conditions, Pollack's team have been able to visualize EZ water inside tunneled gels and hydrophilic tubes made of Nafion immersed in an aqueous microsphere suspension [146] [147].

Videos show a steady flow of microspheres and water along that interior channel, the so-called "self-driven" flow (see also [148]). Light drives that flow; increasing light speeds it by up to five times. Hence, the light driven-flow phenomenon is general; and, it is driven by light. They found that the flow resulted from the protons generated as consequence of EZ growth. Those protons lie in 
the tunnel's central core. Repulsion among protons creates a pressure, which pushes the protons out of one end of the tube or the other. Once that flow begins, additional water gets drawn in from the opposite end of the tube, perpetuating the process.

Pollack's hydroelectric EZ reveal, at least in vitro [141], that IR/UV energy from light outside the organism, and possibly also from biophotons derived from internal exothermic metabolic reactions, may transform simple hydrophilic tubes and vessels, including arteries, veins and capillaries, lymphatic ducts, renal tubules, sweat glands, ureters, tear ducts, Eustachian tubes, respiratory alveolar ducts, bile ducts, cerebrospinal fluid-flow system (CSF-FS), axons ${ }^{12}$, etc., into the equivalent of mechanical pumps but without a motor, i.e. into self-driven liquid-flow systems. That is, all of these tubes and vessels, as well as any other collagen-related liquid-flow system, need pumps to actively move the associated fluids within. The pumping action exerted, e.g. by the cardiac activity on blood and on CSF-FS circulation ${ }^{13}$, as well as the pumping action exerted by the contractile activity of the striated and smooth muscles, behave as pumps and actively move the body fluids, but their efficiency would be drastically reduced without the active contribution of the self-driven liquid-flow system [Figure 5] engaged by proton currents and concentration gradients.

\footnotetext{
${ }^{12}$ Neurons transmit electrical and chemical signals via long slender projections known as axons, neuronal cytoplasmic elongations which enable conduction of electrical impulses along their plasma membranes, as well as chemically mediated signal transduction throughout cytoplasmic organelles, and terminate in synapses. In vertebrates, the axons of many neurons are sheathed in myelin, an EZ-like layer formed by either of two types of glial cells: Schwann cells ensheathing peripheral neurons and oligodendrocytes insulating those of the central nervous system. Axons are hydrophilic tubes that are well suited to structure the water in and around them. Structured water creates an electrical charge that can carry neuro-electrochemical impulses over long distances. The flow of electronic and protonic excitation is the motive force behind nerve transmission.

${ }^{13}$ The cerebral ventricles, the ependymal canal that travels centrally through the spinal cord and the subarachnoid spaces contain the cerebrospinal fluid, produced by the choroid plexuses, which plays a protective function on encephalon and spinal cord interposing between noble and more vulnerable tissues and the skeletal component (skull and spine) which contains them. It is produced continuously, in the healthy adult, in the approximate amount of 305 micro liters per minute and is continuously and passively reabsorbed in the periencephalic spaces with a complete replacement every 6,7 hours. It represents a recent acquisition intended to modify the theories on the pathogenesis of hydrocephalus (dilation of the ventricular system) the fact that the cerebrospinal fluid is reabsorbed partly through the lymphatic system of the nasal mucosa (in fact the cerebral parenchyma does not contain lymphatic vessels) to which it reaches following the subarachnoid spaces that line the olfactory bulbs. The cerebro-spinal liquid flow analysis with Phase Contrast Magnetic Resonance Imaging (PCMRI) [149] [150], shows that the CSF circulation at the level of the ventricular system and of the subarachnoid spaces is a dynamic oscillatory process synchronized with cardiac contractions. To better define this process we can imagine that the brain "pulsates" continuously, driven by cardiac contractions. The changes in blood volume resulting from cardiac contractions determine bi-directional rhythmic movements of cerebrospinal fluid along the cerebral spinal axis: during cardiac systole, the increase in cerebral blood volume determines the cranio-caudal (systolic) displacement of cerebrospinal fluid; instead during diastole the reduction of cerebral blood volume conditions the "diastolic return" of the cerebrospinal fluid in the opposite direction. The described phenomenon is supported by the Monro-Kellie theory [151], according to which the volumetric modifications of brain, blood and cerebrospinal fluid compensate in a dynamic manner (which means that they vary in absolute values but, added together, they always maintain a constant volume equal to that of the bone container).
} 


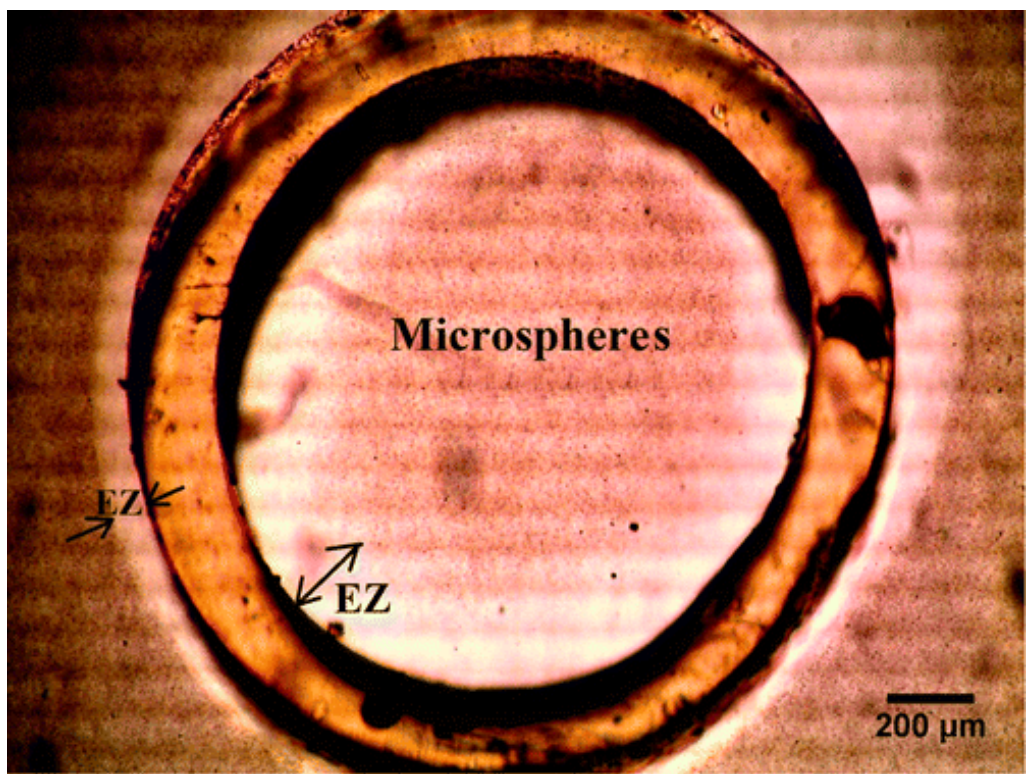

Figure 5. Self-driven flow was observed in Nafion and other hydrophilic tubes immersed in water. The intratubular flow was generated when water came in contact with the tube's hydrophilic surfaces. Flow characteristics were studied in tubes of varying size, exposed to light of different intensities and wavelengths. The results lead to the hypothesis that the flow is driven by a high concentration of protons accumulating inside the tube, creating an axial proton gradient between the inside and outside of the tube. It is also demonstrated a faster flow under incident light, particularly at UV wavelengths, implying that proton generation may be driven by light. Credit [141]. Image source: https://pubs.acs.org/doi/abs/10.1021/la4001945?journalCode=langd5.

If we exclude the mechanical action exerted by the smooth and striated muscles, the main motor force of any biological liquid-flow system is generated across biological membranes and connective-collagen membrane system, that is under EZ/CD electrodynamics laws, by proton currents and concentration gradients.

Thus, the proton currents and concentration gradients generated across biological membranes can be put to physiologic work with cascade effects both at the sub-cellular, cellular, tissue, organ and system levels. The case of self-driven flow system engaged in blood circulation within the blood vesselsis an example: red blood cells flow in the core of vessels and are excluded from the periphery. This feature is long known, and is generally attributed to hydrodynamic effects; but the evidence above implies that it might arise instead from the EZ proton currents and concentration gradients [147].

Blood vessels are made of hydrophilic proteins, which structure the water in blood and form the EZ gel-like state observed at nanoscale in the interaction between water and hydrophilic surface. The negative ions (also in the form of vortical excitations of quasi-free electrons) propel the protons through the middle and drive perfusion. Once the blood plasma is electrically structured, the negative electrical charge, i.e. the zeta potential (the potential that is measured in 
Volts in the process of electrophoresis; if a nanoparticle in a dispersed system moves with electrophoresis to a positive electrode, its charge is negative and vice versa), of red blood vessels builds and prevents them from clustering ${ }^{14}$.

\section{Electromagnetic After-Effect and Keratin Proton Capacitor-Like Effect}

Electromagnetic After-Effect (EAE) and Keratin Proton Capacitor-like Effect (KPC-LE) may be accounted for unusual examples of possible effects linked to connective tissue hydro-electromagnetic stressful related events (exogenous interfacial water stress, EIWS).

Hyper-sensitivity to electromagnetic fields (Electromagnetic Hyper Sensitivity, EHS), also known as electro-sensitivity or Electromagnetic After-Effect (EAE), may be acquired by subjects who have experienced the severely stressful condition due to Near Death State (NDS) or Near Death Like State (NDLS), during which the life-sustaining signs (body temperature, blood pressure, heart rate, respiratory rate and oxygen saturation) are shown to be absent (NDS) or severely altered (NDLS) [153].

EAE manifests itself as the tendency of the subject to interfere with potentially anything electro-magnetic (natural and/or artificial) in the environment (EMEnt). When interference is established, both the Electromagnetic After Effect experiencer (EAEer) and the electro-magnetism of the environment (EMEnt) can be affected. Frequently, EHS is one of a larger complex of sensitivities to which NDSrs (Near Death State experiencers) and NDLSrs (Near Death Like States experiencers) are prone (e.g. allergies, chemical sensitivities, geomagnetic sensitivities). The contextual onset of EHS/EAE in association with other types of sensitivities is manifesting with paresthesia and dysesthesia of various kinds and degrees, and it states for a ground impairment of the mind-body system's (MBS) adaptive feed forward action-reaction towards environmental stimuli (stressors), calling into question, e.g., the integrity of the Psycho-Neuro-Immunological (PNI) system [153].

According to [154] when the interference is established by an apparent action exerted by the EAEer on the EMEnt, we refer to as EM action, inversely, when is established by an apparent action exerted by the EMEnt on the EAEer, we refer to as EM reaction. The deeper the NDS or NDLS it has been, the more likely the Near Death State experiencer (NDSer) or Near Death Like State experience (NDLSer) is to report EM actions and reactions, suggesting that the more severe the stressful action the MBS underwent, the deeper are the consequences suffered by cellular or tissue or organ or system's autopoietic processes.

\footnotetext{
${ }^{14}$ In an ideal system like blood, we want all particulates to have a like electrical charge. If the particles have no electrical charge, the various particles will clump together and form sludge. Therefore, the higher the Zeta Potential, or negative electrical charge, the better the dispersion of particles in suspension. The high Zeta Potential on particles entering the bloodstream may help to increase the dispersion or discreteness of blood cells by helping to enhance the electrical charge on blood colloids which include blood cells (Thomas M. Riddick, 1968) [152].
} 
EAErs can report sensing the presence of EMF in different ways (EM reaction), that is visually (e.g. seeing white light, halos, colors becoming blurry), auditorily (e.g. ringing in the ears, humming, crackling and crunching sounds), kinesthetically (e.g. heart poundings, body vibrating, goose bumps, tingling, physical cringes, little shocks across the body, skin crawling, pressure in the forehead, headaches, hairs raising, floating sensations, shaking hands), interoceptively (e.g. nausea, dizziness, lightheadedness, queasiness, pain, sleeplessness, anxiety, agitation, feeling "drained"), olfactorily (e.g. smelling aromas, a burnt smell, a sweet smell, a menthol breath).

EM actionsmay include:

- computers and other electronics malfunctioning when near;

- lights dimming, flickering, or burning out when near;

- wrist watch batteries die quickly, or watches malfunctioning when worn.

It is here assumed that the severely destabilizing conditions at or near the phase boundary between life and death, which goes to meet the subject experiencing NDS or NDLS, may vary permanently the hydro-electro-magnetic properties of the barrier that separates the inside from the outside of the whole organism, namely the skin, including 1 ) the dielectric constant of the interfacial water (biological water) coherent/incoherent phase (redox pile) of which skin's cells and layers are perfused (EIWS), and, as a consequence 2) epidermis, dermis, hypodermis' dielectric constant (dielectric and electrophysical properties, i.e. dielectrophoresis profile), so to develop the EM actions and/or reactions we observe in EAE.

In particular, it is hypothesized that EHS/EAE may be linked to oxidative stress damage, i.e. the excessive reactive oxygen species (ROS) and reactive nitrogen species (RNS) free radicals mediated damage to cellular macromolecules, to the detriment of connective tissue's chemical and/or hydro-electro-magnetic properties.

Keratin Proton Capacitor-like Effect (KPC-lE) may explain how it can be possible to walk barefoot across a bed of about 482 degrees Celsius (900 degrees Fahrenheit) up to $980^{\circ} \mathrm{C}\left(1.800^{\circ} \mathrm{F}\right)$ burning wood coals (the so-called fire walking, FW) and emerge unscathed.

Conduction is the main way heat is transmitted to a person's feet during a FW. Lousy conductivity of wood followed by the presence of hash on it surface is the first (unconvincing) explanation given by experts to justify how people can walk across a fire pit without getting burned. The second one refers to the possibility of gaining some protective benefit from the so-called Leidenfrost effect ${ }^{15}$, in which a thin layer of sweat or water instantly forms an insulating boundary layer of steam when exposed to intense heat (unfortunately wet feet it carries an added risk of coals sticking to your feet as you walk-increasing exposure time and therefore causing the soles to burn more than if you just crossed with dry feet).

\footnotetext{
${ }^{15}$ See e.g.: https://www.wiley.com/college/phy/halliday320005/pdf/leidenfrost_essay.pdf.
} 
It is here assumed that thanks to a sequence of physical-chemical actions-reactions depending on hydro-electromagnetic and quantum-electrodynamic properties (which include a ten-fold increase in viscosity relative to bulk water, inner negative electric potentials up to $150 \mathrm{mV}$, and, outer concentrations of protons at the boundary between the EZ and bulk water) of keratin intermediate filaments (IF) boundary-water interface confinement, which display, as seen earlier, time-dependent capacitor-like properties, i.e. an excess protons-membrane-excess anions time-dependent capacitor-like system, linked to a proton capacitor concept that can be used to determine the effective localized proton concentrations at the keratin IF boundary-water interface [155], the soles of the feet during FW happen to be thermally-insulated by a $\mathrm{KPC}-\mathrm{lE}$, preventing skin from burning.

\subsection{The Oxidative-Stress-Related NDS/NDLS Electromagnetic Hyper Sensitivity and Electromagnetic After-Effect}

The oxidative-stress-related NDS or NDLS experience may cause a critical, permanent or temporary, alteration to connective tissue's hydro-electromagnetic properties, both at the EZ interfacial water level and at skin's layers level (EIWS), by increasing free radical activity (e.g. via increased free radicals production due to redox cycling reactions), which is known to rest on the free radical pair mechanism, stabilizing free radicals in such a way as to permit their dispersion rather than their return to the ground state, causing NDSr/NDLSr to be anomalously vulnerable (EM reaction) and/orreactive (EM action) to low EMF (L-EMF) exposure.

Free radical pair mechanism involves the recombination of short-lived species, such as reactive free radicals, whose importance in biology and disease is well established [156].

In brief, the free radical pair mechanism requires the creation of free radicals in pairs with correlated electron spins. The thermal and enzyme reactions that produce free radicals in biological systems normally involve singlet states of the precursor molecules. The electrons in the chemical bond that breaks homolytically to form free radicals have antiparallel spins, as do the resulting free radicals themselves. Since the electron spins must be antiparallel to form a bond, the free radicals might be expected to recombine immediately. However, the energy released by the reaction causes them to separate rapidly so that relatively little instantaneous reaction occurs. Subsequently, the magnetic interactions of the electron spins with the nuclei of nearby hydrogen and nitrogen atoms modify the spin state of the radical pair, giving to it partially a triplet character.

The fact that chemical bonds are formed between free radicals with electrons of opposite spin does not mean that the pair of singlet-correlated free radicals produced by homolytic bond splitting would quickly react to form the cage product. Some free radicals do not immediately recombine and because of the released energy they diffuse through their immediate environment. In other words, this is possible because biochemical reactions are not instantaneous but 
depend on overcoming a small activation free energy, or satisfying steric requirements (i.e. a reaction may occur only if the free radicals approach each other in a certain direction). This is crucial for the effect of an EMF to manifest itself on a radical reaction, because it also depends on this rapid initial separation of the formed free radicals.

Most organisms rely on the role of oxygen as a terminal electron acceptor for efficient energy production in the form of ATP. Increased intracellular levels of oxygen, however, are potentially toxic. This toxicity is mainly due to partially reduced forms of $\mathrm{O}_{2}$, since the $\mathrm{O}_{2}$ molecule per se has low reactivity. The molecules and radicals formed by the incomplete reduction of oxygen are termed reactive oxygen species (ROS) and reactive nitrogen species (RNS). ROS/RNS commonly formed in vivo include the superoxide radical anion $\left(\mathrm{O}_{2}{ }^{-}\right)$, hydrogen peroxide $\left(\mathrm{H}_{2} \mathrm{O}_{2}\right)$, and the hydroxyl radical $\left(\mathrm{OH}^{*}\right)$.

ROS/RNS free radicals play important roles in regulation of cell survival. In general, moderate levels of ROS/RNS may function as signals to promote cell proliferation and survival, whereas severe increase of ROS/RNS can induce cell impairment and death. Under physiologic conditions, the balance between generation and elimination of ROS/RNS maintains the proper function of redox-sensitive signaling proteins. Normally, the redox homeostasis ensures that the cells respond properly to endogenous and exogenous stimuli. However, when the redox homeostasis is disturbed, oxidative stress can result in damage to proteins, lipids, nucleic acids, and contribute to disease development.

During normal eukaryote cellular energy synthesis $\mathrm{O}_{2}$ is required by mitochondria (mitochondria are major sources of free radicals [157]) to combine with electrons from the electron transport chain and hydrogen cations from the proton gradient to form water. When $\mathrm{O}_{2}$ is absent during periods of irregular hypoxia in mitochondrial energy synthesis, the generation of excess electrons can develop free radicals or excess protons can produce acid, the latter case being enhanced if ROS hydrogen peroxide $\left(\mathrm{H}_{2} \mathrm{O}_{2}\right)$ produce ROS hydroxyl radical $\left(\mathrm{OH}^{\circ}\right)$ when exposed to transition metal cations such as divalent ferrous iron or $\mathrm{Fe}^{2+}$ common to the heme molecule ${ }^{16}$ and found in connective tissue. Free radicals formed by limited $\mathrm{O}_{2}$ can damage lipids and proteins and greatly increase molecular sizes in growing vicious cycles to reduce oxygen availability even more for mitochondria during energy synthesis. Further, at adequate free-radical concentrations a reactive crosslinking unsaturated aldehyde lipid breakdown product can significantly support free-radical polymerization of lipid oils into rubbery gel-like solids and eventually even produce a crystalline lipid peroxidation with the double bond of $\mathrm{O}_{2}$ [158].

Established causes for temporary or permanent redox homeostasis impair-

\footnotetext{
${ }^{16} \mathrm{Heme}$ proteins are important biological molecules that catalyze radical reactions, and thus they can induce proton spin coupling dependent local field effects on the involved intermediate free radical substrates. Heme proteins are e.g. the important antioxidant enzymes catalases and peroxidases, the oxygen transporters hemoglobin and myoglobin, and all mitochondrial respiratory chain (and photosynthetic electron chain) cytochromes.
} 
ment and increased oxidative stress due to NDS/NDLS related ROS/RNS overproduction, include extended electrolyte imbalance during times of physical crisis, lack of oxygen (hypoxia) all over the body, especially in the brain, or too much carbon dioxide during and after recovery, oxidative injury during cardiopulmonary cerebral resuscitation, hypoglycemia, just to mention a few, all of them causing to release a flood of glutamate, overactivating NMDA (N-methyl-D-aspartate) receptors and resulting in neurotoxicity [159]. Several experiments demonstrated that during post-ischemic reperfusion, there is considerable overproduction of oxygen free radicals generated in different areas [160] [161] [162]. Regarding the energy metabolism, during the hypoxic state, ATP (Adenosine 5'-triphosphate) and glucose exhibit a slight decrease, while ADP (Adenosine 5'-diphosphate) shows an increase that suggests disorders in the tricarboxylic acid cycle (TCA), glycolysis, and mitochondrial oxidative phosphorylation. Fibers of the cytoskeleton conduct electrons from the negative centrosome near the nucleus to the positively charged outer plasma cell membrane surface side as radical negatively charged electrons to provide polymerization chemistry for advancing actin fibers. Electrons conducted through microtubules to actin fibers are generated in excess by mitochondria under irregular oxidative conditions with hypoxia [163].

In the recovery phase, the overproduction of free radicals and excited species can produce significant effects by means of lipid peroxidation [164].

One of the more important electrical properties of the skin is impedance. Electrical impedance is defined as the opposition that show the skin when a current through itself. It is widely accepted that the main electrical impedance resides in the stratum corneum (the uppermost layer of epidermis) while the impedance of the other layers is several orders of magnitudes lower [165].

This resistance is due to the water content of the stratum corneum is very low, not more than $20 \%$, compared to $70 \%$ in the underlying tissue. This means that the skin impedance is dominated by the passive electrical behavior of the stratum corneum and significant differences in impedance values among different anatomical regions of normal skin have been found. The low frequency pathway is dominated by the appendages such as hair follicles and sweat ducts. Lipid lamellae are borderlines between very low conductivity (lipids) and high conductivity (electrolyte) forming a capacitor. There are two distinguishable pathways involving the lipid layers: a direct pathway through the corneocytes and a tortuous pathway using hydrated sites around the corneocytes. Technically, this it can be modeled as a resistor for the appendages and a resistor-capacitor combination for each capacitive pathway in parallel. Since the parameters of the capacitive pathways are distributed, the number of resistor-capacitor combination should be enormous. This combination system showed by the stratum corneum is very reactive and it shows more impedance than resistance. The skin capacitance is a measure of the charge storage capacity of the skin. Therefore, electroporation is known to dramatically change the electrical resistance of lipid-based 
barriers, and cell membranes. More recently, electroporation has been suggested as being responsible for the rapid and large electrical changes that occur because of "high-voltage" pulsing of tissues.

The effect of magnetic fields on free radical reactions [166] primarily originates from the fact that the electron has a magnetic moment because it is electrically charged and has spin angular momentum. Therefore, the electron spin is the electron's EMF angular momentum, making the electron nature's smallest magnet. The electron spin magnetic moment is important in the interaction of atoms with external magnetic fields (MF), in addition to the interaction between the magnetic field and the magnetic dipole moment associated with the electron's orbital angular momentum (due to its rotation around the nucleus). Thus, free radical-involving chemical reactions are affected by an applied MF/EMF because of its interaction with the magnetic moment of the electron [156].

Changes induced under influence of MF [167] may include an increasing refractive index, dielectric constant and electrical conductivity of water with decreasing viscosity, facilitating formation of linear and closed hydrogen-bonded water clusters, the latter of which can become ring electric-current or "molecular electric-current" elements with magnetism due to their proton conductivity under the action of the Lorentz force [168]. This enables (electro)magnetic interactions of these "molecular electric-current" elements with each other or with the externally applied MF/EMF to change the distribution and features of water molecules and the "magnetization of water", which may induce electromagnetic sensitivity and anomalous EMF emission and absorption, followed by EM actions/reactions as observed in EHS/EAE.

However, interaction with external MF/EMFs do not change the nature of the free radical reaction product, but only alter the ratio of free radicals that react in the geminate period ${ }^{17}$ and escape processes, with consequent changes in the ratios of the amounts of cage and escape products. That is, a field may increase the number of escaping free radicals as it is some times observed when free radicals are formed by a homolytic splitting of a singlet state molecule at very low field strengths, including those of the order of the geomagnetic field. Under these conditions, more free radicals survive the geminate period of reaction than at either higher or zero field. This provides a possible mechanism for a field to affect biological processes, given the experimental observation that the increase of oxygen free radicals in organisms is harmful because it imposes to them increased oxidative stress. Although the formation of specific oxygen free radicals under MF/EMF exposure has not yet been shown directly, their indirect pres-

\footnotetext{
${ }^{17}$ When free radicals are produced in solution they are invariably created in pairs. Since small free radicals react with very low activation energies it might be thought that they recombine immediately and no radicals would escape this early, "geminate", period. That they do results from two factors. Firstly the breaking of chemical bonds occurs with release of energy so that the radicals separate. Secondly electron spin orientation is conserved on bond breaking and the radicals produced constitute a "spin correlated radical pair" which possesses the spin multiplicity of their molecular precursor [169].
} 
ence (manifested as oxidative effects on crucial biological molecules such as lipids, DNA, and on the antioxidant defense) has been already documented experimentally [156].

Furthermore, EHS as other sensitivities to which NDSrs and NDLSrs are prone may be also related to Mohri's mechanism of water magnetization [170]. Mohri's hypothesis is based on experimental studies involving physiologically-relevant, $6 \mathrm{~Hz}, 10 \mu \mathrm{T}$ pulsed magnetic field and involves an assumption of cyclotron resonance of protonated water clusters $\left(\mathrm{H}_{3} \mathrm{O}^{+}\left(\mathrm{H}_{2} \mathrm{O}\right) n\right)$. Cyclotron resonance refers to the phenomenon of energy transfer to a charged particle that is moving circularly, normal to the direction of an applied magnetic field, as a manifestation of the Lorentz force; the so-called "cyclotron resonance frequency" of this circular motion depends on the particle's charge and mass and the strength of the magnetic field [1]. According to Mohri, this cyclotron resonance effect activates proton transport in water under the geo-magnetic field, an effect described as "magneto-protonics". Formation of a string of such resonating water clusters can give rise to enhanced proton conductivity. This hypothesis is consistent with the decreased electric resistivity of magnetized water reported by Mohri in studies conducted with weak, pulsed magnetic field [171].

The biological water superstructure constitutes a detailed mold of the biomolecule generating molecular electric dipole interactions in the surrounding water environment. The long range dipole correlations issue by the skin's interfacial water are also acting as carrier waves and may "capture" in their coherent oscillations other neighboring molecules, cells, and biological units by phase-matching. Reciprocally, impurities present in the medium or sources of other kinds, external or endogenous (e.g. free radicals), may operate as de-tuning agents, destroying or altering the coherence of the skin's EZs/CDs, and in a chain of effects, varying 1) the interfacial water dielectric constant [8] [172] [173] [174], 2) that of skin layers, 3) the propagation of the bio-electromagnetic field, with the final result of the appearance of skin pathologies and possibly hypersensitivity to EMF.

In conclusion, critical electro-magnetic/electrodynamic, transient or permanent, variations of skin layers EZs/CDs electronegativity (EIWS) may alter cellular metabolic/energetic regulation systems and the organism's adaptive resources in relation to environmental pressure (it remains to be shown whether the survival capacities of biological systems adapted to extreme environmental conditions are supported by the availability of particular metabolic/energetic regulation systems responsible for EZs electronegativity), to the detriment of organism homeostasis, increasing its vulnerability if electronegativity is lowered (EM reaction), but also with possible anomalous effects if electronegativity is strengthened (EM action).

\subsection{Keratin Proton Capacitor-Like Effect (KPC-IE)}

How is it possible to walk barefoot across a bed of about 482 degrees Celsius 
(900 degrees Fahrenheit) up to $980^{\circ} \mathrm{C}\left(1.800^{\circ} \mathrm{F}\right)$ burning wood coals and emerge unscathed?

The human foot's main function is to facilitate (barefoot)bipedal locomotion, run and upright posture. Contact with the ground during bipedal gait requires a number of complex elastic actions and reactions to absorb and return energy while supporting the application of variable pressure and antigravity propulsive forces. A force is a unit of measure of strength or energy brought to bear on a surface or object, and pressure is the application of force to some object by another object in direct contact with it that is extended over an area. A category of forces known as contact forces affect us in our everyday lives while standing upright, walking and running. The basis for the contact force is the electromagnetic force between atoms. As our foot pushes against the ground, the ground pushes back, propelling us forward. A force of up to one and a half times body weight can be exerted on the human foot at each step while walking, up to three to four times body weight while running and up to eight times while practicing sports. The greatest force is experienced on the forefoot phase of a stride when the whole width of the forefoot absorbs the surge of power that propels the foot off the ground. Over the course of the day, these forces can accumulate up to millions of newtons (hundred or thousands of tons).

The foot is a hypercomplex structure which supports the body and is characterized by being the only body part in contact with the ground, that is, the foot is the "root" between the body and the ground. During gait, movement of the foot is synonymous with movement of all the bones of the lower extremity. An intricate mechanism that cushions the body and adapts to uneven surfaces, the foot provides traction for movement, awareness of joint and body position for balance, and leverage for propulsion. Not only does the foot provide a base of support that allows adjustment to uneven surfaces but also its motion decreases energy expenditure by contributing to the swing phase and stance phase of the gait cycle with dorsiflexion. No surprise if South African palaeoanthropologist P.V. Tobias refers to us as "Man: the tottering biped" [175].

This may explain why human foot represents a highly complex anatomical structure that carries motor-sensor functions and load distribution through a network of nerves, neuromuscular junctions, somatosensory receptors, bones, muscles, myofascial tissue, plantar aponeurosis, joints, tendons, and, last but not least, plantar foot skin, which exhibits unique biophysical properties that are distinct from skin on other areas of the body.

Plantar skin has a unique structure compared to skin on other parts of the body [176]. Its role is to withstand and adapt to the external stresses during (barefoot) physical activities. As a result the epidermis, in particular the stratum corneum (SC), has evolved structural specializations (such as specific spatially arranged keratins) to provide resistance to physical stresses.

Plantar epidermis is known variously as ridged skin, thick skin, glabrous skin or palmoplantar epidermis and it completely lacks hair, has regular arrays of ec- 
crine sweat glands and is highly specialized to resist the high levels of mechanical pressure and trauma encountered in everyday life. The value of $p \mathrm{H}$ for SC is varied depending on SC depth, location and environment and the reported values of SC $p \mathrm{H}$ are in the order of 5 - 7 [177].

Mammalian cells lack any type of exterior cell wall and their strength to resist damage comes from a molecular scaffold, or cytoskeleton, within the cytoplasm. Epithelial cells possess three cytoskeletal systems: actin microfilaments, microtubules and keratin intermediate filaments (IFs) proteins [178]. The actin cytoskeleton is involved in cell attachment and cell migration and the microtubule system is involved in the movement of organelles within the cell.

Keratin IF cytoskeleton is involved in protecting the cell from traumatic damage. Keratins are a large family of IF proteins encoded by 54 distinct genes in humans. About half of these are the epithelial keratins that are found in soft epithelial tissues of the human body. Two keratin polypeptides associate in a parallel arrangement to form an $\approx 50 \mathrm{~nm}$ long coiled coil dimer, consisting of two different types of keratins: one acidic (type I) and one neutral-basic (type II). The two coiled-coil heterodimers further self-assemble into tetramers by packing into an antiparallel half-staggered configuration. Tetramers, in turn, aggregate end-to-end forming protofilaments with a diameter around 2 - $3 \mathrm{~nm}$. Two protofilaments make a protofibril with diameter of order $4-5 \mathrm{~nm}$; four of these assemble laterally to form the keratin IF with diameter of order $8-10 \mathrm{~nm}$. IFs are surrounded by a complex mixture of water, ions, free amino acids and other low molecular weight water soluble non-ionic compounds; this mixture is sometimes referred to as the Natural Moisturising Factor (NMF). NMF plays an important role in skin moisturisation and in maintaining the physico-chemical properties of the skin, such as hydration level, elasticity, permeability and ions availability, and it results from proteolytic degradation of filaggrin, a histidine-rich protein. Schematically, a keratin IF could be pictured as a long cylindrical object filled mainly by $\alpha$-helical coiled coils domains, and decorated on the surface by disordered $\mathrm{N}$ - and C-terminal domains, major component of which is glycine and therefore of hydrophobic nature, extending into the surrounding solution [Figure 6]. Keratins monomers share a common domain structure with other IF proteins. This consists of a central alpha-helical rod domains (negatively charged IF core) of similar substructure $(\approx 310$ amino acids) which is important for polymerization, and two disordered (unstructured) glycine-rich $\mathrm{N}$ - and C-terminal domains (positively charged IF tails) of variable size. Under experimental conditions, $\mathrm{N}$ tails work as the "glue" between IF surfaces while $\mathrm{C}$ tails show much weaker and more long-ranged attraction [177].

Inside the corneocytespositive charge distribution on keratin IF outer surface is expected to take place in the form of a thicker adsorbed layer when uniformly distributed, or adsorb flat on the surface, producing a very thin layer, similar to that of highly charged polyelectrolytes, when clustered [177].

From a quantum-electrodynamic point of view, the positive/negative charge 


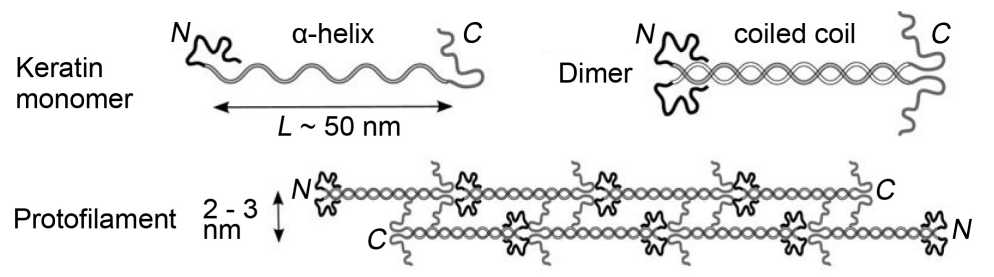

Intermediate Filament (IF)

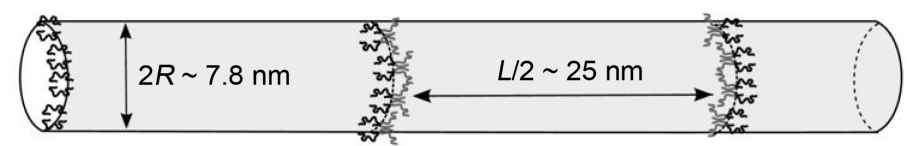

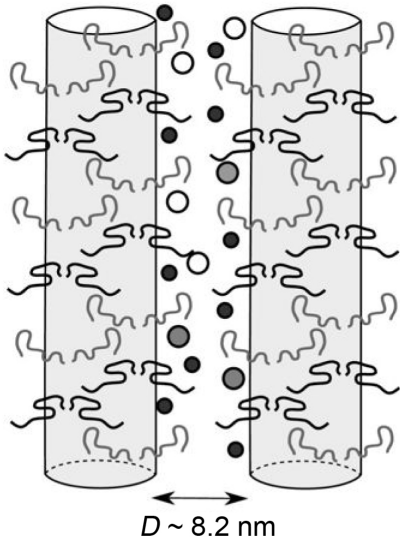

Interactions between two IF

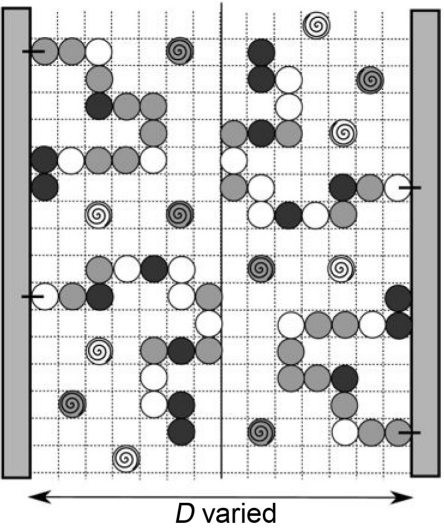

SCF model system for the qap between two IF

Figure 6. IF organisation and the SCF model of N- and C-terminal domains attached onto IF surfaces. The IF surfaces are modelled as plane walls, the grafted domains as connected monomers, the salt ions and/or free amino acids as single monomers. All the other space is occupied by water. The separation between walls is varied in order to obtain the interaction potential mediated by the walls with grafted domains. Credit [177]. Image source: https://bmcbiophys.biomedcentral.com/articles/10.1186/2046-1682-6-12.

distribution on keratin IF wall is equivalent to what we have previously defined excess protons-membrane-excess anions carried on by a time-dependent capacitor-like system.

EZ interfacial water contain ions and display CDs within which magnetic fields can be trapped if the oscillations are optimal. The appropriately patterned extremely low frequency magnetic fields trapped within, results in the emission of specific energies and wavelengths (including laser-like emission) in the visible range [179]. Accordingly, the time-dependent capacitor-like system it may release (contrary to usual assertion about capacitor [180]) an outward (varying) extremely low frequency magnetic field (possibly of up to a $10^{-3}$ gauss order magnetism as shown for palm hands emission [181]), which may underpin a magneto-hydro-dynamic shield-like effect. If this is the case, a suitable increase in the concentration of clustered positive charges (protons) on the keratin IFs outer surface, carried on by the $\mathrm{N}$ - and C-terminal domains, could lead to the 
formation of a thin layer of ionized gas (proton plasma) with insulating properties (thermal insulation), mimicking a thermal shield (magnetic dipole shield), by converting (hydro-magnetic conversion) part of the applied thermal energy to electricity (this could be linked to the sensation of plantar epidermis sizzling, comparable to the effect given by light electric shocks, as occasionally reported by participants after the fire walking ${ }^{18}$ ). The proton-plasma ejected outward by the keratin IF membrane-NMF interface network, possible subjected to ponderomotive forcerepulsion ${ }^{19}$ (a ponderomotive force is a nonlinear force that a "classical" charged particle experiences in an inhomogeneous oscillating electromagnetic field) [100] (see also [185]), would be trapped on the magnetic field lines generated by the EZ/CD excess protons-membrane-excess anions within the keratin IF membrane-NMF time-dependent capacitor-like system, dragging the magnetic field lines out and form a time-dependent skinny-proton plasma insulator.

If confirmed, this metabolic/energetic behavior could be exploited by biological systems, not only via keratin IF membrane-NMF interface network but also via $\mathrm{CD} / \mathrm{EZ}$ membrane-water interface in general, to adapt to occasional and/or permanent exposure to very low or very high thermal gradients. Accordingly, the KPC-lE would be a special case of this adaptive strategy and the plantar epidermis in contact with the glowing wood would be (at least partially) protected from burning by the temporary formation of a skinny-proton plasma insulator on its outer surface.

But this hypothesis would be incomplete, anyway, if we did not take into account the fact that fire walking is a ceremony, belonging to extreme collective rituals. This is, the fire walking was born as a collective ritual and always takes place, apart from a few show-like performances, in a collective form ${ }^{20}$. As in

\footnotetext{
${ }^{18}$ In this regard Italian anthropologist Lorenzo de Martino [182] cites the experience of fire walking performed during the Umu-Ti ceremony, in Rarotonga (Cook archipelago), by the ethnologist W. E. Gudgeon [183], who writes (my translation): I did not walk quickly through the furnace, but cautiously, because I was afraid to put my foot on some sharp points of the stones, and then to fall. My feet also were very delicate. I forgot to tell you that during the crossing of the furnace I had the impression that my feet were completely peeling. Moreover, when the crossing was completed, I felt a tingling similar to light electric shocks under the soles of my feet, and this lasted for seven hours or more.

${ }^{19}$ The basis of a time-averaged ponderomotive forceis that electromagnetic waves transfer energy and momentum to matter. Intuitively, wave-induced ponderomotive forces are expected to be repulsive, i.e. the "heaviness" (in Latin ponderos) of waves provides a transfer of energy and momentum in the direction of wave propagation [184]. However, ponderomotive forces are more complicated, and have consequences that may contradict intuition. An example of this is magnetic moment pumping, MMP, a ponderomotive force in magnetized plasmas with diverging magnetic fields. The MMP force is always in the direction of the magnetic field divergence-regardless of wave propagation direction and wave frequency. The MMP force exemplifies that the term "heaviness" can be a misleading. The gradient/Miller force is a more striking exception to the intuitive concept of "heaviness" of waves. The direction of the Miller force inplasmas critically depends on the local resonance frequency, e.g. the ion gyrofrequency, $\Omega$. For Alfvén waves in magnetized plasma, the Miller force is repulsive at frequencies above-, and attractive at frequencies below $\Omega$. The Miller force therefore agrees with intuition on wave momentum exchange only in the high-frequency domain.

${ }^{20}$ Even when performed by middle-class whites searching for extreme (quasi-miraculous) experiences: Finally, I argue that the mechanism that helps white, middle-class Americans breach logical convention and participate in firewalking and related rituals is a form of mutual pretense, a social ritual engaged to shield people from bleak realities and graceless moments [186].
} 
other collective ritual forms (indeed, this is their power), every single member of the community, or group, directly (fire walker) or indirectly (supporter and spectator) involved in the fire walking ceremony, tend to merge into a single subject (at least this should be the condition for any collective ritual to be effective), in a spiritual, emotional, psychological, physical, tensional catharsis, which may induce individuals to lose their identity to become part of an integrated anthropo-poietic system, characterized by a collective choral, undivided, coherent behavior. When this happens, a phase transition, pointing at a collective phase-matching, takes place, and the community becomes a super-coherence domain, whose dynamics, from a QED point of view, can be traced back to those of a Bose-Einstein condensate ${ }^{21}$.

As early mentioned (see footnote $n^{\circ}$ ), external electromagnetic signals can be selectively damped by tissues (in the present case by the integrated anthropo-poietic system), according to their being or not in phase with the possible oscillatory motion of the system's components. This specific phase-matching (resonance or coupling phase) feature operates as a very selective mechanism, a sort of filter discriminating among perturbations and stimuli acting on the system, thus protecting it against any noisy perturbative background or even strong actions, which, however, are out of phase with the oscillatory motions allowed by the system's inner dynamics.

Accordingly, the phase transition to a state of oscillatory super-coherence, namely the passage via phase $\Phi$ sharing from a single coherenceathropo-domain with its $\Phi$ to a collective coherenceathropo-domain with $\Phi f(\mathrm{x})$ (coherence between single coherence athropo-domain), increases the adaptive capacities of the system, making it less permeable to destabilizing or harmful external stimuli. This increased capacity for self-defense and self-repair can affect all the physiological processes set up for it, including the processes pertaining to the KPC-lE. The result would be an increased protective function of the latter during fire walking.

\section{Conclusions}

The exploration of the aquatic environment at abyssal depths may reserve some astonishing surprises, such as the presence of microbes and animals that flourish in extreme environmental conditions (such as complete darkness and no photosynthesis may occur, food-limited environment, high hydrostatic pressures up to 300 - $500 \mathrm{MPs}$, compared to the $0.1 \mathrm{MPs}$ atmospheric pressure measured at sea surface level). This is the case of the discovery of the lush communities of animals inextricably tied to microbes that live around sites of active hydrothermal venting along oceanic spreading centers [187] [188]. A wide diversity of bacteria

\footnotetext{
${ }^{21}$ Phase transitions to unitary, coordinated and coherent behavior are ubiquitous in all biological environments and are reminiscent of that observed in fish shoals, flocks of birds, communities of ants and termites, swarms of bees, capable of maneuvering in dynamic three-dimensional geometric forms that mimic the evolution of strange attractors in non-linear chaotic systems (deterministic chaos).
} 
can colonize the habitats bathed in fluids with temperatures below $150^{\circ} \mathrm{C}$, and animals often live in temperatures near $60^{\circ} \mathrm{C}$. In 2003 , was reported the isolation of hyperthermophilic archaeon strain 121 from the Juan de Fuca Ridge (Northeast Pacific Ocean), growing at temperatures up to $121^{\circ} \mathrm{C}$, which is still the highest growth temperature documented for microbial life. Metazoans (multicellular animals) often colonize the surfaces of hydrothermal chimneys and are much more restricted with respect to temperature tolerance than microbes. However, the highest-biomass assemblages of animals are usually found in areas of lower temperature, diffuse flow (ambient to about $40^{\circ} \mathrm{C}$ ) emitted through cracks in the basalt or andesite substrate. It has been demonstrated that the sulfide worm Paralvinellasulfincola tolerates body temperatures between $50^{\circ} \mathrm{C}$ and $55^{\circ} \mathrm{C}$, the highest demonstrated for a marine metazoan. Equally extreme and challenging aspects of the vent environment that animals have adapted to are the thermal gradients, both in space and in time. Animals like the chimney dwellers live in an environment where one end of their bodies can be exposed to waters near $60^{\circ} \mathrm{C}$ while the other end is in water below $10^{\circ} \mathrm{C}$, sometimes even in the ambient deep-sea temperatures near $2^{\circ} \mathrm{C}$. These gradients must present significant physiological challenges to metazoans, which must maintain physiological homeostasis, while many molecules and processes are drastically affected by temperature over this range. Although there is abundant oxygen in the ambient bottom water over most hydrothermal vents, many of the microhabitats occupied by animals at vents contain little or no oxygen most of the time. To facilitate survival in these low-oxygen habitats, the majority of the larger animals have oxygen-binding proteins with much higher affinities for oxygen than their close relatives from more benign environments. Although habitats with relatively high and variable temperature, and high-sulfide and low-oxygen concentrations present the biggest challenge to biological differentiation, these are also the habitats with the highest potential rewards for the fauna that can adapt to them. These are the areas where chemoautotrophic bacteria will thrive, producing a rich food source, and also the areas where predation pressure may be the least (as predators must also be specially adapted to tolerate these habitats, and remarkably few appear to have evolved these adaptations). Because chemoautotrophic bacteria can thrive at extremes that animals cannot tolerate, it should come as no surprise that hydrothermal vent fauna have evolved to push the limits of metazoan tolerance to oxygen, temperature, and sulfide.

The first deep-sea hydrothermal vent ecosystem was discovered a mere 40 years ago (1977) and several key aspects of even the best-studied animals are not yet well understood. It would be very interesting to verify if, and to what extent, the QED approach can give any help in understanding the biochemical and biophysical aspects of these organisms' physiology. I believe it can.

\section{Conflicts of Interest}

The author declares no conflict of interest. 


\section{References}

[1] Davidson, R.M., Lauritzen, A. and Seneff, S. (2013) Biological Water Dynamics and Entropy: A Biophysical Origin of Cancer and Other Diseases. Entropy, 15, 3822-3876. https://people.csail.mit.edu/seneff/Entropy/entropy-15-03822.pdf https://doi.org/10.3390/e15093822

[2] Preparata, G., et al. (1999) The Role of QED (Quantum Electro Dynamics) in Medicine. Rivista di Biologia/Biology Forum 93/ 2000, 1-27.

http://www.22passi.it/downloads/biorisonanza/qeddefinitivo.pdf

[3] Pérez, C., et al. (2012) Structures of Cage, Prism, and Book Isomers of Water Hexamer from Broadband Rotational Spectroscopy. Science, 336, 897-901.

https://www.researchgate.net/publication/224979747_Structures_of_Cage_Prism_a nd_Book_Isomers_of_Water_Hexamer_from_Broadband_Rotational_Spectroscopy https://doi.org/10.1126/science.1220574

[4] Ho, M.W. (2014) Large Supramolecular Water Clusters Caught on Camera-A Review. Water Journal, 6, 1-12. https://www.waterjournal.org/volume-6/ho

[5] Jerman, I. and Ratajc, P. (2014) A Further Indication of Self-Ordering Capacity of Water via the Droplet Evaporation Method. Entropy, 16, 5211-5222.

https://www.mdpi.com/1099-4300/16/10/5211 https://doi.org/10.3390/e16105211

[6] Elia, V., et al. (2013) Experimental Evidence of Stable Aggregates of Water at Room Temperature and Normal Pressure after Iterative Contact with a Nafion ${ }^{\circledR}$ Polymer Membrane. Water Journal, 5, 16-26.

http://www.waterjournal.org/volume-5/de-ninno

[7] Bischof, M. and Del Giudice, E. (2013) Communication and the Emergence of Collective Behavior in Living Organisms: A Quantum Approach. Molecular Biology International, 2013, Article ID: 987549. https://doi.org/10.1155/2013/987549 http://www.oalib.com/paper/3079684\#.XJyuG7ieErs

[8] Tigrek, S. and Barnes, F. (2010) Water Structures and Effects of Electric and Magnetic Fields. In: Giuliani, L. and Soffritti, M., Eds., Non-Thermal Effects and Mechanisms of Interaction between Electromagnetic Fields and Matter, European Journal of Oncology, 25-50. http://www.teslabel.be/PDF/ICEMS_Monograph_2010.pdf

[9] Carignano, M.A., Karlström, G. and Linse, P. (1997) Polarizable Ions in Polarizable Water: A Moleclar Dynamics Study. Journal of Physical Chemistry B, 101, 1142-1147.

[10] Enami, S. and Colussi, A.J. (2013) Long-Range Specific Ion-Ion Interactions in Hydrogen-Bonded Liquid Films. The Journal of Chemical Physics, 138, Article ID: 184706. https://doi.org/10.1063/1.4803652 https://pdfs.semanticscholar.org/1917/fadacf2b318da1d31b95c489483dd33ab30d.pdf

[11] Rahman, N.A., Ibrahim, F. and Yafouz, B. (2017) Dielectrophoresis for Biomedical Sciences Applications: A Review. Sensors, 17, 449.

https://www.ncbi.nlm.nih.gov/pmc/articles/PMC5375735/ https://doi.org/10.3390/s17030449

[12] Pethig, R., Menachery, A., Pells, S. and De Sousa, P. (2010) Dielectrophoresis: A Review of Applications for Stem Cell Research. Journal of Biomedicine and Biotechnology, 2010, Article ID: 182581. https://doi.org/10.1155/2010/182581 https://www.researchgate.net/publication/44618612_Dielectrophoresis_A_Review_ of_Applications_for_Stem_Cell_Research

[13] Dukhin, A.S., Ulberg, Z.R., Gruzina, T.G. and Karamushka, V.I. (2014) Peculiarities 
of Live Cells' Interaction with Micro- and Nanoparticles. In: Ohshima, H. and Makino, K., Eds., Colloid and Interface Science in Pharmaceutical Research and Development, Elsevier, Amsterdam, 193-222. https://doi.org/10.1016/B978-0-444-62614-1.00010-7

[14] Du, E., Qiang, Y. and Liu, J. (2018) Erythrocyte Membrane Failure by Electromechanical Stress. Applied Sciences, 8, 174. https://doi.org/10.3390/app8020174 https://www.ncbi.nlm.nih.gov/pmc/articles/PMC5909407/

[15] Ho, M.W. (2014) Illuminating Water and Life. Entropy, 16, 4874-4891. https://www.mdpi.com/1099-4300/16/9/4874/pdf https://doi.org/10.3390/e16094874

[16] Reiter, G.F., et al. (2012) Evidence for an Anomalous Quantum State of Protons in Nanoconfined Water. Physical Review, 85, Article ID: 045403.

https://arxiv.org/abs/1101.4994 https://doi.org/10.1103/PhysRevB.85.045403

[17] Gilli, G. and Gilli, P. (2012) Six Lectures on the Nature of the Hydrogen Bond, Lecture 1, Introduction to the Hydrogen Bond: Basic Concepts and Summary of Our First Studies from 1989 to 2002. http://www.ggilli.com/files/2012_1_INTRODUCTION.pdf

[18] Gilli, P. and Gilli, G. (2010) Hydrogen Bond Models and Theories: The Dual Hydrogen Bond Model and Its Consequences. Journal of Molecular Structure, 972, 2-10. https://doi.org/10.1016/j.molstruc.2010.01.073

[19] Gilli, G. and Gilli, P. (2009) The Nature of the Hydrogen Bond: Outline of a Comprehensive Hydrogen Bond Theory. Oxfrord University Press, Oxfrord. https://doi.org/10.1093/acprof:oso/9780199558964.001.0001

[20] Ceriotti, M., Cuny, J., Parrinello, M. and Manolopoulos, D.E. (2013) Nuclear Quantum Effects and Hydrogen Bond Fluctuations in Water. Proceedings of the National Academy of Sciences of the United States of America, 110, 15591-15596. https://www.pnas.org/content/110/39/15591 https://doi.org/10.1073/pnas.1308560110

[21] Hutter, J. (2012) Car-Parrinello Molecular Dynamics. Wiley Interdisciplinary Reviews. Computational Molecular Science, 2, 604-612. https://doi.org/10.1002/wcms.90

[22] Lock, A., Woutersen, S. and Bakker, H.J. (2001) Ultrafast Energy Equilibration in Hydrogen-Bonded Liquids. The Journal of Physical Chemistry A, 105, 1238-1243. https://doi.org/10.1021/jp003158e

[23] Woutersen, S., Emmerichs, U., Nienhuys, H.-K. and Bakker, H.J. (1998) Anomalous Temperature Dependence of Vibrational Lifetimes in Water and Ice. Physical Review Letters, 81, 1106-1109. https://doi.org/10.1103/PhysRevLett.81.1106 http://hankwang.lagom.nl/publications/woutersen-prl-81-1106-1998.pdf

[24] Deàk, J., Rhea, S.T., Iwaki, L.K. and Dlott, D.D. (2000) Vibrational Energy Relaxation and Spectral Diffusion in Water and Deuterated Water. The Journal of Physical Chemistry $A, 104,4866-4875$.

[25] Tuladhar, A., Dewan, S., Kubicki, J.D. and Borguet, E. (2016) Spectroscopy and Ultrafast Vibrational Dynamics of Strongly Hydrogen Bonded $\mathrm{OH}$ Species at the $\alpha-\mathrm{Al}_{2} \mathrm{O}_{3}(11 \overline{2} 0) / \mathrm{H}_{2} \mathrm{O}$ Interface. The Journal of Physical Chemistry $C, 120$, 16153-16161. http://www.temple.edu/borguet/publication/Documents/pdf_files/2016-2-si.pdf

[26] Woutersen, S., Emmerichs, U. and Bakker, H.J. (1997) Femtosecond Mid-IR Pump-Probe Spectroscopy of Liquid Water: Evidence for a Two-Component 
Structure. Science, 278, 658-660. https://doi.org/10.1126/science.278.5338.658 http://citeseerx.ist.psu.edu/viewdoc/download?doi=10.1.1.894.3843\&rep=rep1\&type $=\mathrm{pdf}$

[27] Laenen, R., Rauscher, C. and Laubereau, A. (1998) Dynamics of Local Substructures in Water Observed by Ultrafast Infrared Hole Burning. Physical Review Letters, 80, 2622-2625. https://doi.org/10.1103/PhysRevLett.80.2622

[28] Woutersen, S. and Bakker, H.J. (1999) Hydrogen Bond in Liquid Water as a Brownian Oscillator. Physical Review Letters, 83, 2077-2080.

https://www.researchgate.net/profile/Sander_Woutersen/publication/243475463_H ydro-

gen_Bond_in_Liquid_Water_as_a_Brownian_Oscillator/links/54ee22b10cf25238f93 9b514.pdf https://doi.org/10.1103/PhysRevLett.83.2077

[29] Sendner, C., Horinek, D., Bocquet, L. and Netz, R.R. (2009) Interfacial Water at Hydrophobic and Hydrophilic Surfaces: Slip, Viscosity, and Diffusion. Langmuir, 25, 10768-10781. https://doi.org/10.1021/la901314b https://www.academia.edu/16943925/Interfacial_Water_at_Hydrophobic_and_Hyd rophilic_Surfaces_Slip_Viscosity_and_Diffusion

[30] Patel, A.J., et al. (2012) Sitting at the Edge: How Biomolecules Use Hydrophobicity to Tune Their Interactions and Function. The Journal of Physical Chemistry B, 116, 2498-2503. https://www.ncbi.nlm.nih.gov/pmc/articles/PMC3303187/ https://doi.org/10.1021/jp2107523

[31] Messori, C. (2019) The Super-Coherent State of Biological Water. Open Access Library Journal, 6, 1-5. https://www.scirp.org/journal/PaperInformation.aspx?PaperID=90862

[32] Davidson, R.M. and Seneff, S. (2012) The Initial Common Pathway of Inflammation, Disease, and Sudden Death. Entropy, 14, 1399-1442.

https://doi.org/10.3390/e14081399 https://dspace.mit.edu/openaccess-disseminate/1721.1/76366

[33] Preoteasa, E.A. and Apostol, M.V. (2008) Collective Dynamics of Water in the Living Cell and in Bulk Liquid. New Physical Models and Biological Inferences, International Fröhlich Symposium Biophysical Aspects of Cancer Electromagnetic Mechanisms, Prague, Czech Republic. https://arxiv.org/ftp/arxiv/papers/0812/0812.0275.pdf

[34] Sigel, R. (2017) Concepts for Soft Interfaces. Soft Matter, 13, 1940-1942. https://pubs.rsc.org/en/content/articlepdf/2017/sm/c6sm02413k https://doi.org/10.1039/C6SM02413K

[35] Pokorný, J., et al. (2015) Mitochondrial Dysfunction and Disturbed Coherence: Gate to Cancer. Pharmaceuticals, 8, 675-695. https://doi.org/10.3390/ph8040675 https://www.ncbi.nlm.nih.gov/pmc/articles/PMC4695805/

[36] Pokorný, J., Pokorný, J. and Borodavka, F. (2017) Warburg Effect-Damping of Electromagnetic Oscillations. Electromagnetic Biology and Medicine, 36, 270-278. https://emmind.net/openpapers_repos/Endogenous_Fields-Mind/General/EM_Can cer/2017_Warburg_effect_damping_of_electromagnetic_oscillations.pdf https://doi.org/10.1080/15368378.2017.1326933

[37] Pokorný, J. (2014) Cancer-Pathological Breakdown of Coherent Energy States. Biophysical Reviews and Letters, 9, 115-133.

https://www.researchgate.net/profile/Jan_Vrba/publication/263805231_CANCER-P Athologi- 
cal_breakdown_of_coherent_energy_states/links/57207e6308aed056fa236c4d.pdf?di $\underline{\text { sableCoverPage }=\text { true }}$ https://doi.org/10.1142/s1793048013300077

[38] Antonenko, Y.N., Pohl, P. and Rosenfeld, E. (1996) Visualization of the reaction layer in the Immediate Membrane Vicinity. Archives of Biochemistry and Biophysics, 333, 225-232. https://doi.org/10.1006/abbi.1996.0385

[39] Del Giudice, E. and Vitiello, G. (2011) Influence of Gravity on the Collective Molecular Dynamics of Liquid Water: The Case of the Floating Water Bridge. Water Journal, 2, 133-141. http://waterjournal.org/uploads/vol2/delgiudice/WATER.2011.1.Vitiello.pdf

[40] Del Giudice, E., Stefanini, P., Tedeschi, A. and Vitiello, G. (2011) The Interplay of Biomolecules and Water at the Origin of the Active Behavior of Living Organisms. Journal of Physics. Conference Series, 329, Article ID: 012001. https://iopscience.iop.org/article/10.1088/1742-6596/329/1/012001/pdf https://doi.org/10.1088/1742-6596/329/1/012001

[41] Del Giudice, E., Pulselli, R.M. and Tiezzi, E. (2009) Thermodynamics of Irreversible Processes and Quantum Field Theory: An Interplay for the Understanding of Ecosystem Dynamics. Ecological Modelling, 220, 1874-1879.

http://www.irafs.org/courses/materials/delgiudice_thermodynamics.pdf https://doi.org/10.1016/j.ecolmodel.2009.04.035

[42] Brizhik, L., Del Giudice, E., Jørgensen, S.E., Marchettini, N. and Tiezzi, E. (2009) The Role of Electromagnetic Potentials in the Evolutionary Dynamics of Ecosystems. Ecological Modelling, 220, 1865-1869.

https://www.researchgate.net/publication/222525342_The_role_of_electromagnetic _potentials_in_the_evolutionary_dynamics_of_ecosystems https://doi.org/10.1016/j.ecolmodel.2009.04.017

[43] Brizhik, L., Del Giudice, E., Tedeschi, A. and Voeikov, V.L. (2011) The Role of Water in the Information Exchange between the Components of an Ecosystem. Ecological Modelling, 222, 2869-2877. https://doi.org/10.1016/j.ecolmodel.2011.05.017 https://www.researchgate.net/publication/229327908_The_role_of_water_in_the_in formation_exchange_between_the_components_of_an_ecosystem

[44] Brizhik, L., Chiappini, E., Stefanini, P. and Vitiello, G. (2018) Modeling Meridians within the Quantum Field Theory. Journal of Acupuncture and Meridian Studies, 12, 29-36. https://www.jams-kpi.com/article/S2005-2901(18)30076-1/pdf

[45] Messori, C. (2016) From Continuity to Contiguity: On the Genesis of Consciousness, Culture and Oral Language (Part IV of IV). Journal of Consciousness Exploration \& Research, 7, 214-228.

https://www.researchgate.net/publication/298791037_From_Continuity_to_Contiguity On_the_genesis_of_consciousness_culture_and_oral_language_Part_IV_of_IV

[46] Murugan, N.J., Karbowski, L.M. and Persinger, M.A. (2014) Serial pH Increments ( 20 to 40 Milliseconds) in Water during Exposures to Weak, Physiologically Patterned Magnetic Fields: Implications for Consciousness. Water Journal, 6, 45-60. http://www.waterjournal.org/volume-6/persinger-summary-2

[47] Fumagalli, L., et al. (2018) Anomalously Low Dielectric Constant of Confined Water. Science, 360, 1339-1342. https://arxiv.org/ftp/arxiv/papers/1806/1806.04486.pdf https://doi.org/10.1126/science.aat4191

[48] Voeikov, V.L. and Del Giudice, E. (2009) Water Respiration-The Basis of the Living State. Water Journal, 1, 52-75. https://www.waterjournal.org/uploads/vol1/voeikov/WATER-Vol1-Voeikov.pdf 
[49] Tedeschi, A. (2010) Is the Living Dynamics Able to Change the Properties of Water? International Journal of Design \& Nature Ecodynamics, 5, 60-67.

https://www.witpress.com/Secure/ejournals//papers/D\&NE050108f.pdf https://doi.org/10.2495/DNE-V5-N1-60-67

[50] Montagnier, L., et al. (2015) Transduction of DNA Information through Water and Electromagnetic Waves. Electromagnetic Biology and Medicine, 34, 106-112.

https://arxiv.org/abs/1501.01620 https://doi.org/10.3109/15368378.2015.1036072

[51] Wernet, P.H., Nordlund, D., Bergmann, U. and Cavalleri, M. (2004) The Structure of the First Coordination Shell in Liquid Water. Science, 304, 995-998.

https://doi.org/10.1126/science.1096205 https://www.researchgate.net/publication/8641681_The_Structure_of_the_First_Co ordination_Shell_in_Liquid_Water

[52] Voeikov, V.L., et al. (2012) The Stable Nonequilibrium State of Bicarbonate Aqueous Systems. Russian Journal of Physical Chemistry A, 86, 1407-1415.

https://www.researchgate.net/publication/257845152_The_stable_nonequilibrium_s tate_of_bicarbonate_aqueous_systems https://doi.org/10.1134/S003602441209018X

[53] Bernadi, L., Valle, F., Coco, M., Calciati, A. and Sleight, P. (1996) Physical Activity Influences Heart Rate Variability and Very-Low-Frequency Components in Holter Electrocardiograms. Cardiovascular Research, 32, 234-237.

https://doi.org/10.1016/0008-6363(96)00081-8

[54] McCraty, R., Deyhle, A. and Childre, D. (2012) The Global Coherence Initiative: Creating a Coherent Planetary Standing Wave. Global Advances in Health and Medicine, 1, 64-76. https://www.ncbi.nlm.nih.gov/pmc/articles/PMC3833489/ https://doi.org/10.7453/gahmj.2012.1.1.013

[55] Kleimenova, N.G., Kozyreva, O.V., Breus, T.K. and Rapoport, S.I. (2007) Pc1 Geomagnetic Pulsations as a Potential Hazard of the Myocardial Infarction. Journal of Atmospheric and Solar-Terrestrial Physics, 69, 1759-1764.

https://doi.org/10.1016/j.jastp.2006.10.018

https://www.researchgate.net/publication/223464840_Pc1_geomagnetic_pulsations as_a_potential_hazard_of_the_myocardial_infarction

[56] Samsonov, S.N., Kleimenova, N.G., Kozyreva, O.V. and Petrova, P.G. (2014) The Effect of Space Weather on Human Heart Diseases in Subauroral Latitudes. Izvestiya Atmospheric and Oceanic Physics, 59, 719-727.

https://doi.org/10.1134/S0001433814040057

https://www.researchgate.net/publication/284401991_The_effect_of_space_weather _on_human_heart_diseases_in_subauroral_latitudes

[57] Meyl, K. (2012) About Vortex Physics and Vortex Losses. Journal of Vortex Science and Technology, 1, Article ID: 235563. https://doi.org/10.4303/jvst/235563

http://omicsonline.com/open-access/about-vortex-physics-and-vortex-losses-20908369.1000101.pdf?aid=15110

[58] Del Giudice, E. and Tedeschi, A. (2009) Water and the Autocatalysis in Living Matter. Electromagnetic Biology and Medicine, 28, 46-54. http://www.ncbi.nlm.nih.gov/pubmed/19337894 https://doi.org/10.1080/15368370802708728

[59] Szent-Gyorgyi, A. (1957) Bioenergetics. Academic Press, New York.

[60] Del Giudice, E., Voeikov, V., Tedeschi, A. and Vitiello, G. (2015) The Origin and 
Special Role of Coherent Water in Living Systems. In: Fels, D., Cifra, M. and Scholkmann, F., Eds., Fields of the Cell, Research Signpost, Trivandrum, 95-111. https://emmind.net/openpapers_repos/Endogenous_Fields-Mind/Water_EMF/Excl usion_Zones/2014_The_origin_and_the_special_role_of_coherent_water_in_living systems.pdf

[61] Meyl, K. (2012) DNA and Cell Resonance: Magnetic Waves Enable Cell Communication. DNA and Cell Biology, 31, 422-426.

https://doi.org/10.1089/dna.2011.1415

http://www.k-meyl.de/go/Primaerliteratur/Magnetic_Waves-Enable-Cell_Commun ication.pdf

[62] Pollack, G.H. (2013) The Fourth Phase of Water: Beyond Solid, Liquid, and Vapor. Edgescience, No. 16, 14-18.

https://ecee.colorado.edu/ ecen5555/SourceMaterial/Pollack13.pdf

[63] Pollack, G.H., Figueroa, X. and Zhao, Q. (2009) Molecules, Water, and Radiant Energy: New Clues for the Origin of Life. International Journal of Molecular Sciences, 10, 1419-1429. https://doi.org/10.3390/ijms10041419

https://www.ncbi.nlm.nih.gov/pmc/articles/PMC2680624/

[64] Hwang, S.G., Hong, J.K., Sharma, A., Pollack, G.H. and Bahng, G.W. (2018) Exclusion Zone and Heterogeneous Water Structure at Ambient Temperature. PLoS ONE, 13, e0195057. https://doi.org/10.1371/journal.pone.0195057 https://journals.plos.org/plosone/article?id=10.1371/journal.pone.0195057

[65] Del Giudice, E., Tedeschi, A., Vitiello, G. and Voeikov, V. (2013) Coherent Structures in Liquid Water Close to Hydrophilic Surfaces. Journal of Physics. Conference Series, 442, Article ID: 012028. https://doi.org/10.1088/1742-6596/442/1/012028 https://iopscience.iop.org/article/10.1088/1742-6596/442/1/012028/pdf

[66] Zheng, J.M. and Pollack, G.H. (2003) Long-Range Forces Extending from Polimer-Gel Surfaces. Physical Review E: Statistical Nonlinear and Soft Matter Physics, 68, 031408-031414. https://doi.org/10.1103/PhysRevE.68.031408 https://arxiv.org/ftp/cond-mat/papers/0305/0305093.pdf

[67] Zheng, J.M., Chin, W.-C., Khijniak, E., Khijniak Jr., E. and Pollack, G.H. (2006) Surfaces and Interfacial Water: Evidence that Hydrophilic Surfaces Have Long-Range Impact. Advances in Colloid and Interface Science, 127, 19-27.

https://doi.org/10.1016/j.cis.2006.07.002 http://courses.washington.edu/bioe555/Zheng.pdf

[68] Chai, B., et al. (2008) Spectroscopic Studies of Solutes in Aqueous Solution. The Journal of Physical Chemistry A, 112, 2242-2247.

[69] Buch, V., Tarbuck, T., Groenzin, H., Li, I., Shultz, M.J. and Richmond, G.L. (2007) Sum Frequency Generation Surface Spectra of Ice, Water, and Acid Solution Investigated by an Exciton Model. The Journal of Chemical Physics, 127, Article ID: 204710. https://doi.org/10.1063/1.2790437 https://pages.uoregon.edu/grgroup/Publications/157\%20Buch.pdf

[70] Del Giudice, E., Spinetti, P.R. and Tedeschi, A. (2010) Water Dynamics at the Root of Metamorphosis in Living Organisms. Water Journal, 2, 566-586. https://doi.org/10.3390/w2030566 https://pdfs.semanticscholar.org/4bc0/3fdbd99780f6713d375a74bd9200d525b2a7.pdf

[71] Palti, Y., de Nour, E. and Abrahamov, A. (1966) The Effect of Atmospheric Ions on the Respiratory System of Infants. Pediatrics, 38, 405-411.

[72] Kotaka, S. and Krueger, A.P. (1978) Effects of Air Ions on Microorganisms and Other Biological Materials. Critical Reviews in Microbiology, 6, 109-150. 
https://doi.org/10.3109/10408417809090621

[73] Charry, J.M. and Hawkinshire, F.B. (1981) Effects of Atmospheric Electricity on Some Substrates of Disordered Social Behavior. Journal of Personality and Social Psychology, 41, 185-197. https://doi.org/10.1037//0022-3514.41.1.185

[74] Bachman, C.H., McDonald, R.D. and Lorenz, P.J. (1965) Some Physiological Effects of Measured Air Ions. International Journal of Biometeorology, 9, 127-139. https://doi.org/10.1007/BF02188468

[75] SovijÄrvi, A.R.A., et al. (1979) Effect of Air Ionization on Heart Rate and Perceived Exertion during a Bicycle Exercise Test. European Journal of Applied Physiology and Occupational Physiology, 41, 285-291. https://doi.org/10.1007/BF00429745

[76] Charry, J.M. (1984) Biological Effects of Small Air Ions: A Review of Findings and Methods. Environmental Research, 34, 351-389. https://doi.org/10.1016/0013-9351(84)90104-X

[77] Luts, A., et al. (2009) Some Air Electricity Phenomena Caused by Waterfalls: Correlative Study of the Spectra. Atmospheric Research, 91, 229-237. https://doi.org/10.1016/j.atmosres.2008.02.019

[78] Kurt Kung, C.-C. and Pollack, G.H. (2014) Effect of Atmospheric Ions on Interfacial Water. Entropy, 16, 6033-6041. https://doi.org/10.3390/e16116033 https://www.mdpi.com/1099-4300/16/11/6033

[79] Jennie, P., Mather, J.P. and Roberts, P.E. (1998) Introduction to Cell and Tissue Culture Theory and Technique. Plenum Press, New York. https://www.bjcancer.org/Sites_OldFiles/_Library/UserFiles/pdf/Introduction\%20to \%20Cell\%20and\%20Tissue\%20Culture.pdf

[80] Ullmann, G.M. and Bombarda, E. (2013) pKa Values and Redox Potentials of Proteins. What Do They Mean? Biological Chemistry, 394, 611-619.

http://www.bisb.uni-bayreuth.de/PDF/Ullmann2013.BiolChem.pdf https://doi.org/10.1515/hsz-2012-0329

[81] Darcy, J.W., Koronkiewicz, B., Parada, G.A. and Mayer, J.M. (2018) A Continuum of Proton-Coupled Electron Transfer Reactivity. Accounts of Chemical Research, 51, 2391-2399. https://doi.org/10.1021/acs.accounts.8b00319

http://gaznevada.iq.usp.br/wp-content/uploads/2018/10/mayer-18_PCET_continuu m.pdf

[82] Weinberg, D.R., et al. (2007) Proton-Coupled Electron Transfer. Chemical Reviews, 107, 5004-5064. https://www.ncbi.nlm.nih.gov/pmc/articles/PMC3449329/ https://doi.org/10.1021/cr0500030

[83] Sbitnev, V.I. (2016) Quantum Consciousness in Warm, Wet, and Noisy Brain. Modern Physics Letters B, 30, Article ID: 1650329. https://arxiv.org/abs/1606.00258 https://doi.org/10.1142/S0217984916503292

[84] Silvi, B. and Ratajczak, H. (2016) Hydrogen Bonding and Delocalization in the ELF Analysis Approach. Physical Chemistry Chemical Physics, 18, 27442-27449.

https://doi.org/10.1039/C6CP05400E https://hal.sorbonne-universite.fr/hal-01383126/document

[85] Decoursey, T.E. (2003) Voltage-Gated Proton Channels and Other Proton Transfer Pathways. Physiological Reviews, 83, 475-579.

https://www.physiology.org/doi/full/10.1152/physrev.00028.2002?url_ver=Z39.88-2 003\&rfr_id=ori:rid:crossref.org\&rfr_dat=cr_pub\%3dpubmed

[86] Pomès, R. and Roux, B. (1998) Free Energy Profiles for $\mathrm{H}^{+}$Conduction along Hydrogen-Bonded Chains of Water Molecules. Biophysic Journal, 75, 33-40. 
https://doi.org/10.1016/S0006-3495(98)77492-2

https://www.ncbi.nlm.nih.gov/pmc/articles/PMC1299677/

[87] Hassanali, A., Giberti, F., Cuny, J., Kühne, T.D. and Parrinello, M. (2013) Proton Transfer Through the Water Grossamer. Proceedings of the National Academy of Sciences of the United States of America, 110, 13723-13728. https://doi.org/10.1073/pnas.1306642110

[88] Peng, Y., Swanson, J.M.J., Kang, S., Zhou, R. and Voth, G.A. (2015) Hydrated Excess Protons Can Create their Own Water Wires. Journal of Physical Chemistry $B, 119,9212-9218$. https://pubs.acs.org/doi/10.1021/jp5095118

[89] Mollenhauer, H.H. and Morré, D.J. (1978) Structural Compartmentation of the Cytosol: Zones of Exclusion, Zones of Adhesion, Cytoskeletal and Intercisternal Elements. In: Roodyn, D.B., Ed., Subcellular Biochemistry, Springer, Boston, MA, 327-362. https://doi.org/10.1007/978-1-4615-7942-7_7

[90] Miller Jr., J.H., Rajapakshe, K.I., Infante, H.L. and Claycomb, J.R. (2013) Electric Field Driven Torque in ATP Synthase. PLoS One, 8 e74978.

https://doi.org/10.1371/journal.pone.0074978

https://journals.plos.org/plosone/article?id=10.1371/journal.pone.0074978

[91] Messori, C. (2012) A Cosmogonic Model of Human Consciousness. Journal of Consciousness Exploration \& Research, 3, 1149-1208.

https://www.researchgate.net/publication/255696726_A_Cosmogonic_Model_of_H uman_Consciousness

[92] Fedi, M. (2017) Hydrodynamics of the Dark Superfluid: I. Genesis of Fundamental Particles. Hal-01549082. https://hal.archives-ouvertes.fr/hal-01549082v2/document

[93] Fedi, M. (2016) Quantum Gravity without Gravitons in a Superfluid Quantum Space. Hal-01362019. https://hal.archives-ouvertes.fr/hal-01362019/document

[94] Fedi, M. (2017) A Superfluid Theory of Everything? Hal-01312579v4. https://hal.archives-ouvertes.fr/hal-01312579v4/document

[95] Dellago, C., Naor, M.M. and Hummer, G. (2003) Proton Transport through Water-Filled Carbon Nanotubes. Physical Review Letters, 90, Article ID: 105902. https://doi.org/10.1103/PhysRevLett.90.105902 https://homepage.univie.ac.at/Christoph.Dellago/papers/PRL_90_105902_2003.pdf

[96] Chen, J., Li, X.-Z., Zhang, Q., Michaelides, A. and Wang, E. (2013) Nature of Proton Transport in a Water-Filled Carbon Nanotube and in Liquid Water. Physical Chemistry Chemical Physics, 15, 6344-6349. https://doi.org/10.1039/c3cp50218j https://arxiv.org/abs/1404.7280

[97] Zhu, F. and Schulten, K. (2003) Water and Proton Conduction through Carbon Nanotubes as Models for Biological Channels. Biophysical Journal, 85, 236-244. https://doi.org/10.1016/S0006-3495(03)74469-5 https://www.sciencedirect.com/science/article/pii/S0006349503744695

[98] Little, W.A. (1964) Possibility of Synthesizing an Organic Superconductor. Physical Review, 134, A1416-A1424. https://doi.org/10.1103/PhysRev.134.A1416 http://ivanik3.narod.ru/SuperCondactivyty/Hot/PhysRev.134.A1416Littei.pdf

[99] Fainchtein, R. (1992) Scanning Tunneling Microscopy of Organic Conductors and Superconductors. Johns Hopkins APL Technical Digest, 13, 332-341. https://www.jhuapl.edu/techdigest/views/pdfs/V13_N2_1992/V13_N2_1992_Fainch tein.pdf

[100] Del Giudice, E. and Giuliani, L. (2010) Coherence in Water and the kT Problem in Living Matter. In: Giuliani, L. and Soffritti, M., Eds., Non-Thermal Effects and Me- 
chanisms of Interaction between Electromagnetic Fields and Matter, European Journal of Oncology, 5(Monograph), 7-23.

http://www.teslabel.be/PDF/ICEMS_Monograph_2010.pdf

[101] Kresin, V.Z. (2018) Paths to Room-Temperature Superconductivity. Journal of Superconductivity and Novel Magnetism, 31, 611-617.

https://doi.org/10.1007/s10948-017-4382-0

[102] Sahu, S., Ghosh, S., Hirata, K., Fujita, D. and Bandyopadhyay, A. (2013) Multi-Level Memory-Switching Properties of a Single Brain Microtubule. Applied Physics Letters, 102, Article ID: 123701. https://doi.org/10.1063/1.4793995 https://www.researchgate.net/profile/Anirban_Bandyopadhyay/publication/257953 778_Multi-level_memory-switching_properties_of_a_single_brain_microtubule/lin ks/02e7e526f888007a08000000/Multi-level-memory-switching-properties-of-a-singl e-brain-microtubule.pdf

[103] Sahu, S., et al. (2013) Atomic Water Channel Controlling Remarkable Properties of a Single Brain Microtubule: Correlating Single Protein to Its Supramolecular Assembly. Biosensors and Bioelectronics, 47, 141-148.

https://doi.org/10.1016/j.bios.2013.02.050

[104] Vasiliev, B.V. (2013) Superconductivity and Superfluidity-Part I: The Development of the Science of Superconductivity and Superfluidity in the 20th Century. Universal Journal of Physics and Application, 1, 392-407. http://www.hrpub.org/download/20131201/UJPA5-18400592.pdf

[105] Vasiliev, B.V. (2014) Superconductivity and Superfluidity-Part II: Superconductivity as a Consequence of Ordering of Zero-Point Oscillations in Electron Gas. Universal Journal of Physics and Application, 2, 22-35. http://www.hrpub.org/download/20131215/UJPA6-18400592II.pdf

[106] Kaplana, D. and Imry, Y. (2018) High-Temperature Superconductivity Using a Model of Hydrogen Bonds. Proceedings of the National Academy of Sciences of the United States of America, 115, 5709-5713. https://doi.org/10.1073/pnas.1803767115 https://www.ncbi.nlm.nih.gov/pmc/articles/PMC5984540/

[107] Mann, A. (2011) High-Temperature Superconductivity at 25: Still in Suspense. Nature, 475, 280-282. https://doi.org/10.1038/475280a https://www.nature.com/news/2011/110720/full/475280a.html\#B4

[108] Pines, D. (2002) The Spin Fluctuation Model for High Temperature Superconductivity: Progress and Prospects. In: Bock, J., Deutscher, G., Pavuna, D. and Wolf, S.A., Eds., The Gap Symmetry and Fluctuations in High-Tc Superconductors, NATO Science Series: B, Springer, Boston, MA, 111-142.

https://doi.org/10.1007/0-306-47081-0_7

https://www.scribd.com/document/94654905/486d283e69-Bok-Supercond

[109] Fedi, M. (2017) Gravity as a Fluid Dynamic Phenomenon in a Superfluid Quantum Space. Fluid Quantum Gravity and Relativity. Hal-01248015v4. https://hal.archives-ouvertes.fr/hal-01248015v4

[110] Sbitnev, V.I. (2018) Hydrodynamics of Superfluid Quantum Space: De Broglie Interpretation of the Quantum Mechanics. Quantum Studies. Mathematics and Foundations, 5, 257-271. https://arxiv.org/abs/1707.08508 https://doi.org/10.1007/s40509-017-0116-Z

[111] Reiter, G.F., et al. (2011) Evidence of a New Quantum State of Nano-Confined Water. Mesoscale and Nanoscale Physics, ArXiv: 1101.4994. https://arxiv.org/abs/1101.4994

[112] Bakker, H.J. and Nienhuys, H.-K. (2002) Delocalization of Protons in Liquid Water. 
Science, 297, 587-590. https://doi.org/10.1126/science.1073298

https://pdfs.semanticscholar.org/ce35/61432c26353604d79ef6f12f5a119fbefaf3.pdf

[113] Del Giudice, E., Preparata, G. and Vitiello, G. (1988) Water as a Free Electric Dipole Laser. Physical Review Letters, 61, 1085-1088.

https://doi.org/10.1103/PhysRevLett.61.1085

https://www.researchgate.net/publication/13249471_Water_as_a_Free_Electric_Dip ole_Laser

[114] Arani, R., Bono, I., Del Giudice, E. and Preparata, G. (1995) QED Coherence and the Thermodynamics of Water. International Journal of Modern Physics B, 9 , 1813-1841. https://doi.org/10.1142/S0217979295000744

[115] Chen, B., Ivanov, I., Klein, M.L. and Parrinello, M. (2003) Hydrogen Bonding in Water. Physical Review Letters, 91, Article ID: 215503.

https://doi.org/10.1103/PhysRevLett.91.215503

https://www.researchgate.net/profile/Ivaylo_Ivanov4/publication/8953214_Hydroge n_Bonding_in_Water/links/0c960533dcdd1e9efc000000.pdf

[116] Benoit, M., Marx, D. and Parrinello, M. (1998) Tunnelling and Zeropoint Motion in High Pressure Ice. Nature, 392, 258-261. https://doi.org/10.1038/32609 https://www.researchgate.net/publication/224015851_Tunnelling_and_zero-point_ motion_in_high-pressure_ice

[117] Pamuk, B., et al. (2012) Anomalous Nuclear Quantum Effects in Ice. Physical Review Letters, 108, Article ID: 193003.

https://doi.org/10.1103/PhysRevLett.108.193003

https://repositorio.uam.es/bitstream/handle/10486/668161/Anomalous_Pamuk_prL 2012.pdf? sequence $=1$

[118] Tuckerman, M.E., Marx, D. and Parrinello, M. (2002) The Nature and Transport Mechanism of Hydrated Hydroxide Ions in Aqueous Solution. Nature, 417, 925-929. https://doi.org/10.1038/nature00797

https://www.researchgate.net/publication/11288822_The_Nature_and_Transport_ Mechanism_of_Hydrated_Hydroxide_Ions_in_Aqueous_Solution

[119] Paesani, F. and Voth, G.A. (2008) Quantum Effects Strongly Influence the Surface Premelting of Ice. Journal of Physical Chemistry C Letters, 112, 324-327. https://doi.org/10.1021/jp710640e

[120] Nagata, Y., Pool, R.E., Backus, E.H.G. and Bonn, M. (2012) Nuclear Quantum Effects Affect Bond Orientation of Water at the Water-Vapor Interface. Physical Review Letters, 109, 226101-226105. https://doi.org/10.1103/PhysRevLett.109.226101 https://www.researchgate.net/publication/235389075_Nuclear_Quantum_Effects_A ffect_Bond_Orientation_of_Water_at_the_Water-Vapor_Interface

[121] Liu, J., et al. (2013) A Surface-Specific Isotope Effect in Mixtures of Light and Heavy Water. Journal of Physical Chemistry C, 117, 2944-2951. https://doi.org/10.1021/jp311986m

[122] Markland, T. and Berne, B. (2012) Unraveling Quantum Mechanical Effects in Water Using Isotopic Fractionation. Proceedings of the National Academy of Sciences of the United States of America, 109, 7988-7991.

https://doi.org/10.1073/pnas.1203365109

https://www.pnas.org/content/109/21/7988

[123] Ye, H., Naguib, N. and Gogotsi, Y. (2004) TEM Study of Water in Carbon Nanotubes. JEOL News, 39, 38-43.

https://www.researchgate.net/publication/267646721_TEM_Study_of_Water_in_Ca rbon_Nanotubes 
[124] Naguib, N., et al. (2004) Observation of Water Confined in Nanometer Channels of Closed Carbon Nanotubes. NanoLetters, 4, 2237-2243. https://doi.org/10.1021/nl0484907

[125] Hassanali, A.A., Cuny, J., Ceriotti, M., Pickard, C.J. and Parrinello, M. (2012) The Fuzzy Quantum Proton in the Hydrogen Chloride Hydrates. Journal of the American Chemical Society, 134, 8557-8569. https://doi.org/10.1021/ja3014727

[126] Horbatenko, Y. and Vyboishchikov, S.F. (2011) Hydrogen Motion in Proton Sponge Cations: A Theoretical Study. Chemphyschem, 12, 1118-1129. https://doi.org/10.1002/cphc.201000721

[127] Bienko, A., Bieńko, A.J., Latajka, Z., Sawka-Dobrowolska, W. and Sobczyk, L. (2003) Low Barrier Hydrogen Bond in Protonated Proton Sponge. X-Ray Diffraction, Infrared, and Theoretical $a b$ initio and Density Functional Theory Studies. The Journal of Chemical Physics, 119, 4313-4319. https://doi.org/10.1063/1.1594171

[128] Li, X.-Z., Probert, M.I.J., Alavi, A. and Michaelides, A. (2010) Quantum Nature of the Proton in Water-Hydroxyl Overlayers on Metal Surfaces. Physical Review Letters, 104, Article ID: 066102. https://doi.org/10.1103/PhysRevLett.104.066102 https://www.ucl.ac.uk/catalytic-enviro-group/wp-content/uploads/2016/03/Li-Prob ert-Alavi-Michaelides-PRL2010.pdf

[129] Bothma, J., Gilmore, J. and McKenzie, R.H. (2010) The Role of Quantum Effects in Proton Transfer Reactions in Enzymes: Quantum Tunneling in a Noisy Environment? New Journal of Physics, 12, Article ID: 055002.

https://doi.org/10.1088/1367-2630/12/5/055002 https://iopscience.iop.org/article/10.1088/1367-2630/12/5/055002/pdf

[130] Grewer, C., Gameiro, A., Mager, T. and Fendler, K. (2013) Electrophysiological Characterization of Membrane Transport Proteins. Annual Review of Biophysics, 42, 95-120. https://doi.org/10.1146/annurev-biophys-083012-130312

[131] Watanabe, R., et al. (2014) Arrayed Lipid Bilayer Chambers Allow Single-Molecule Analysis of Membrane Transporter Activity. Nature Communications, 5, Article No. 4519. https://doi.org/10.1038/ncomms5519 https://www.nature.com/articles/ncomms5519

[132] Gutiérrez-Sanz, Ó., et al. (2015) Induction of a Proton Gradient across a Gold-Supported Biomimetic Membrane by Electroenzymatic $\mathrm{H}_{2}$ Oxidation. Angewandte Chemie International Edition, 54, 2684-2687.

[133] Mitchell, P. (1961) Coupling of Phosphorylation to Electron and Hydrogen Transfer by a Chemi-Osmotic Type of Mechanism. Nature, 191, 144-148. https://doi.org/10.1038/191144a0

[134] Arnold, R.S., et al. (2009) Mitochondrial DNA Mutation Stimulates Prostate Cancer Growth in Bone Stromal Environment. The Prostate, 69, 1-11. https://www.ncbi.nlm.nih.gov/pmc/articles/PMC2753601/

[135] Shidara, Y., et al. (2005) Positive Contribution of Pathogenic Mutations in the Mitochondrial Genome to the Promotion of Cancer by Prevention from Apoptosis. Cancer Research, 65, 1655-1663. https://doi.org/10.1158/0008-5472.CAN-04-2012 http://cancerres.aacrjournals.org/content/65/5/1655

[136] Mattiazzi, M., et al. (2004) The mtDNA T8993G (NARP) Mutation Results in an Impairment of Oxidative Phosphorylation that Can Be Improved by Antioxidants. Human Molecular Genetics, 13, 869-879. https://doi.org/10.1093/hmg/ddh103 https://academic.oup.com/hmg/article/13/8/869/2355757

[137] Sgarbi, G., et al. (2006) Inefficient Coupling between Proton Transport and ATP Synthesis May Be the Pathogenic Mechanism for NARP and Leigh Syndrome Re- 
sulting from the T8993G Mutation in mtDNA. Biochemical Journal, 395, 493-500. https://www.researchgate.net/publication/7368871_Inefficient_coupling_between_pro ton_transport_and_ATP_synthesis_may_be_the_pathogenic_mechanism_for_NAR

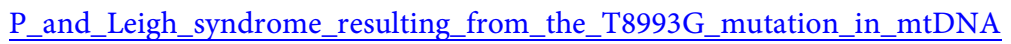
https://doi.org/10.1042/BJ20051748

[138] Simon, J., van Spanning, R.J.M. and Richardson, D.J. (2008) The Organization of Proton Motive and Non-Proton Motive Redox Loops in Prokaryotic Respiratory Systems. Biochimica et BiophysicaActa, 1777, 1480-1490.

https://doi.org/10.1016/j.bbabio.2008.09.008

https://www.sciencedirect.com/science/article/pii/S0005272808006695

[139] Saeed, H.A. and Lee, J.W. (2018) Experimental Determination of Proton-Cation Exchange Equilibrium Constants at Water-Membrane Interface Fundamental to Bioenergetics. Water Journal, 9, 116-140.

http://www.waterjournal.org/volume-9/lee

[140] Georgievskii, Y., Medvedev, E.S. and Stuchebrukhov, A.A. (2002) Proton Transport via Coupled Surface and Bulk Diffusion. Journal of Chemical Physics, 116, 1692-1699. https://doi.org/10.1063/1.1428350

[141] Rohani, M. and Pollack, G.H. (2013) Flow through Horizontal Tubes Submerged in Water in the Absence of a Pressure Gradient: Mechanistic Considerations. Langmuir, 29, 6556-6561. https://doi.org/10.1021/la4001945

[142] Ho, M.W., Zhou, Y.-M., Haffegee, J. and Watton, A. (2006) The Liquid Crystalline Organism and Biological Water. In: Pollack, G., Ed., Water in Cell Biology, Springer, Dordrecht.

https://www.researchgate.net/publication/226563146_The_Liquid_Crystalline_Orga nism_and_Biological_Water

[143] Bardelmeyer, G.H. (1973) Electrical Conduction in Hydrated Collagen. I. Conductivity Mechanisms. Biopolymers, 12, 2289-2302.

https://doi.org/10.1002/bip.1973.360121008

[144] Stoller, P.C., et al. (2003) Effects of Structural Modification on Second Harmonic Generation in Collagen. Proceedings of SPIE 4963, Multiphoton Microscopy in the Biomedical Sciences III, 41-51.

https://www.spiedigitallibrary.org/conference-proceedings-of-spie/4963/1/Effect-of-struct ural-modification-on-second-harmonic-generation-in-collagen/10.1117/12.477998.short

[145] Reise, K., Stoller, P. and Knoesen, A. (2017) Three-Dimensional Geometry of Collagenous Tissues by Second Harmonic Polarimetry. Scientific Reports, 7, Article No. 2642. https://doi.org/10.1038/s41598-017-02326-7 https://www.ncbi.nlm.nih.gov/pmc/articles/PMC5453962/

[146] Pollack, G.H. (2017) Why Hydrogels Don't Dribble Water. Gels, 3, 43. https://doi.org/10.3390/gels3040043 https://www.mdpi.com/2310-2861/3/4/43/pdf

[147] Yu, A., Carlson, P. and Pollack, G.H. (2014) Unexpected Axial Flow through Hydrophilic Tubes: Implications for Energetics of Water. The European Physical Journal Special Topics, 223, 947-958. https://doi.org/10.1140/epjst/e2013-01837-8

[148] Qiana, S. and Bau, H.H. (2009) Magneto-Hydrodynamics Based Microfluidics. Mechanics Research Communications, 36, 10-21.

https://doi.org/10.1016/j.mechrescom.2008.06.013 https://www.ncbi.nlm.nih.gov/pmc/articles/PMC2768299/

[149] Spijkerman, J.M., et al. (2018) Phase Contrast MRI Measurements of Net Cerebros- 
pinal Fluid Flow through the Cerebral Aqueduct Are Confounded by Respiration. Journal of Magnetic Resonance Imaging, 49, 433-444.

https://doi.org/10.1002/jmri.26181

https://onlinelibrary.wiley.com/doi/pdf/10.1002/jmri.26181

[150] Balédent, O., et al. (2006) Value of Phase Contrast Magnetic Resonance Imaging for Investigation of Cerebral Hydrodynamics. Journal of Neuroradiology, 33, 292-303.

https://doi.org/10.1016/S0150-9861(06)77287-X

https://www.em-consulte.com/en/article/126927

[151] Kim, D.-J., et al. (2012) Continuous Monitoring of the Monro-Kellie Doctrine: Is It Possible? Journal of Neurotrauma, 29, 1354-1363.

https://doi.org/10.1089/neu.2011.2018

https://www.ncbi.nlm.nih.gov/pmc/articles/PMC3335107/

[152] Riddick, T.M. (1968) Control of Colloid Stability through Zeta Potential: With a Closing Chapter on Its Relationship to Cardiovascular Disease. Livingston Publishing Company, London.

[153] Messori, C. (2018) Near Death Experiences: Falling Down a Very Deep Well. Open Access Library Journal, 5, e4985. https://doi.org/10.4236/oalib.1104985 http://www.oalib.com/articles/5300585\#.XJoQ1LieErs

[154] Blalock, S. and Atwater, P.M.H. (2016) Electromagnetic and Other Environmental Effects Following Near-Death Experiences: A Primer. Journal of Near-Death Studies, 33, 181-211. https://doi.org/10.17514/JNDS-2015-33-4-p181-211.

[155] Lee, J.W. (2012) Proton-Electrostatics Hypothesis for Localized Proton Coupling Bioenergetics. Bioenergetics, 1, 1-8.

https://www.google.it/url?sa=t\&rct=j\&q=\&esrc=s\&source=web\&cd=1\&cad=rja\&ua $\mathrm{ct}=8 \& v e d=2$ ahUKEwjHsdDwt7HiAhUKx4UKHaKSCp8QFjAAegQIBRAC\&url=htt ps\%3A\%2F\%2Fwww.longdom.org\%2Fopen-access\%2Fprotonelectrostatics-hypothe sis-for-localized-protoncoupling-bioenergetics-2167-7662.1000104.pdf\&usg=AOvV aw2awAFlHh1h-WRJ056wzkNy

[156] Georgiou, C.D. (2010) Oxidative Stress-Induced Biological Damage by Low-Level EMFs: Mechanism of Free Radical Pair Electron Spin Polarization and Biochemical Amplification. In: Giuliani, L. and Soffritti, M., Eds., Non-Thermal Effects and Mechanisms of Interaction between Electromagnetic Fields and Matter, European Journal of Oncology, 5 (Monograph), 63-113.

http://www.teslabel.be/PDF/ICEMS_Monograph_2010.pdf

[157] Thar, R. and Kühl, M. (2004) Propagation of Electromagnetic Radiation in Mitochondria? Journal of Theoretical Biology, 230, 261-270.

https://doi.org/10.1016/j.jtbi.2004.05.021

https://www.researchgate.net/publication/8407688_Propagation_of_electromagneti c_radiation_in_mitochondria

[158] Petersen, R.C., Reddy, M.S. and Liu, P.-R. (2018) Advancements in Free-Radical Pathologies and an Important Treatment Solution with a Free-Radical Inhibitor. $S F$ Journal of Biotechnology and Biomedical Engineering, 1, 1-13. https://scienceforecastoa.com/Articles/SJBBE-V1-E1-1003.pdf

[159] Jansen, K.L.R. (1997) The Ketamine Model of the Near-Death Experience: A Central Role for the N-Methyl-D-Aspartate Receptor. Journal of Near-Death Studies, 16, 5-26. https://doi.org/10.17514/JNDS-1997-16-1-p63-69.

[160] Agardh, C.D., Zhang, H., Smith, M.-L. and Siesjö, B.K. (1991) Free Radical Production and Ischemic Brain Damage: Influence of Postischemic Oxygen Tension. International Journal of Developmental Neuroscience, 9, 127-138. 
https://doi.org/10.1016/0736-5748(91)90003-5

[161] Ophir, A., et al. (1993) Hydroxyl Radical Generation in the Cat Retina during Reperfusion Following Ischemia. Experimental Eye Research, 57, 351-357. https://doi.org/10.1006/exer.1993.1134

[162] Basu, S., Miclescu, A., Sharma, H. and Wiklund, L. (2011) Propofol Mitigates Systemic Oxidative Injury during Experimental Cardiopulmonary Cerebral Resuscitation. Prostaglandins, Leukotrienes and Essential Fatty Acids, 84, 123-130. https://doi.org/10.1016/j.plefa.2010.11.006

[163] Petersen, R.C. (2013) Free-Radical Polymer Science Structural Cancer Model: A Review. Scientifica, 2013, Article ID: 143589. https://doi.org/10.1155/2013/143589 https://www.hindawi.com/journals/scientifica/2013/143589/

[164] Suzuki, J., Imaizumi, S., Kayama, T. and Yoshimoto, T. (1985) Chemiluminescence in Hypoxic Brain-The Second Report: Cerebral Protective Effect of Mannitol, Vitamin E and Glucocorticoid. Stroke, 16, 695-700.

https://doi.org/10.1161/01.STR.16.4.695

https://pdfs.semanticscholar.org/1523/3f7d88a401eb12dc06b7bc6fdddb20e52a15.pdf

[165] Lu, F., et al. (2018) Review of Stratum Corneum Impedance Measurement in Non-Invasive Penetration Application. Biosensors, 8, 31.

https://doi.org/10.3390/bios8020031 https://www.ncbi.nlm.nih.gov/pmc/articles/PMC6023082/

[166] Wang, H. and Zhang, X. (2017) Magnetic Fields and Reactive Oxygen Species. International Journal of Molecular Sciences, 18, Article No. 2175.

https://doi.org/10.3390/ijms18102175 https://www.mdpi.com/1422-0067/18/10/2175/htm

[167] Pang, X.F. and Deng, B. (2008) The Changes of Macroscopic Features and Microscopic Structures of Water under Influence of Magnetic Field. Physica B: Condensed Matter, 403, 3571-3577. https://doi.org/10.1016/j.physb.2008.05.032 http://www.deltawater.net/wp-content/uploads/2017/08/1.3.pdf

[168] Pang, X.F. (2006) The Conductivity Properties of Protons in Ice and Mechanism of Magnetization of Liquid Water. The European Physical Journal B: Condensed Matter and Complex Systems, 49, 5-23. https://doi.org/10.1140/epjb/e2006-00020-6

[169] Eveson, R.W. and McLauchlan, K.A. (1999) Electron Spin Polarization Studies of the Dynamics of Geminate Free Radical Reactions. RIKEN Review, No. 24, 25-27. http://www.dl.ndl.go.jp/view/download/digidepo_8428544_po_24_025.pdf?content $\underline{\mathrm{No}=10 \& \text { alternativeNo }}$

[170] Mohri, K. and Fukushima, M. (2003) Milligauss Magnetic Field Triggering Reliable Self-Organization of Water with Long-Range Ordered Proton Transport through Cyclotron Resonance. IEEE Transactions on Magnetics, 39, 3328-3330. https://doi.org/10.1109/TMAG.2003.816766

[171] Mohri, K., et al. (2010) Sensing of Human Microvibration Transmitted along Solid Using Pico-Tesla Magneto-Impedance Sensor (pT-MI Sensor). PIERS Online, 6, 161-164. http://www.piers.org/piersonline/pdf/Vol6No2Page161to164.pdf

[172] Bonthuis, D.J., Uematsu, Y. and Netz, R.R. (2015) Interfacial Layer Effects on Surface Capacitances and Electro-Osmosis in Electrolytes. Philosophical Transactions of the Royal Society A: Mathematical, Physical and Engineering Sciences, 374. https://doi.org/10.1098/rsta.2015.0033 http://rsta.royalsocietypublishing.org/content/374/2060/20150033

[173] Bonthuis, D.J. (2014) Dielectric Profiles and Ion-Specific Effects at Aqueous Inter- 
faces. Electrostatics of Soft and Disordered Matter, 129-142.

http://www-thphys.physics.ox.ac.uk/people/DouweBonthuis/documents/2014_Bont huis_bookchapter.pdf

[174] Teschke, O., Ceotto, G. and de Souza, E.F. (2001) Interfacial Water Dielectric-Permittivity-Profile Measurements Using Atomic Force Microscopy. Physical Review E, 64, Article ID: 011605. https://doi.org/10.1103/PhysRevE.64.011605 http://repositorio.unicamp.br/bitstream/REPOSIP/60423/1/WOS000169907100061. pdf

[175] Tobias, P.V. (1982) Man: The Tottering Biped: The Evolution of his Posture, Poise and Skill. CPME, Kensington.

[176] Swensson, O., et al. (1998) Specialized Keratin Expression Pattern in Human Ridged Skin as an Adaptation to High Physical Stress. British Journal of Dermatology, 139, 767-775. https://doi.org/10.1046/j.1365-2133.1998.02499.x

[177] Akinshina, A., Jambon-Puillet, E., Warren, P.B. and Noro, M.G. (2013) Self-Consistent Field Theory for the Interactions between Keratin Intermediate Filaments. BMC Biophysics, 6, 12. https://doi.org/10.1186/2046-1682-6-12 https://bmcbiophys.biomedcentral.com/articles/10.1186/2046-1682-6-12

[178] Bragulla, H.H. and Homberger, D.G. (2009) Structure and Functions of Keratin Proteins in Simple, Stratified, Keratinized and Cornified Epithelia. Journal of Anatomy, 214, 516-559. https://doi.org/10.1111/j.1469-7580.2009.01066.x https://www.ncbi.nlm.nih.gov/pmc/articles/PMC2736122/[181]

[179] Del Giudice, E. and Preparata, G. (1994) Coherent Dynamics in Water as a Possible Explanation of Biological Membranes Formation. Journal of Biological Physics, 20, 105-116.

[180] Papachristou, C. (2018) Some Remarks on the Charging Capacitor Problem. Advanced Electromagnetics, 7, 10-12. https://doi.org/10.7716/aem.v7i2.694 https://arxiv.org/ftp/arxiv/papers//1802/1802.01652.pdf

[181] Seto, A., et al. (1992) Detection of Extraordinary Large Bio-Magnetic Field Strengh from Human Hand during External Qi Emission. Acupuncture \& Electro-Therapeutic Research, 17, 75-94. https://doi.org/10.3727/036012992816357819

[182] De Martino, L. (2017) Il Mondo Magico. Bollati Boringhieri Publisher, Turin, 21.

[183] Gudgeon, W.E. (1899) The Umi-Ti, or Fire-Walking Ceremony. The Journal of the Polynesian Society, VIII.

[184] Lundin, R., Lidgren, H. (2010) On the Attraction of Matter by the Ponderomotive Miller Force. Plasma Physics, ArXiv: 1005.4913. https://arxiv.org/abs/1005.4913

[185] Hora, H. (2016) Introduction to the Ponderomotion Processes and Overview of Related Phenomena. In: Hora, H., Ed., Laser Plasma Physics. Forces and the Nonlinearity Principle, SPIE Digital Library. https://spie.org/samples/PM250.pdf

[186] Edwards, E.D. (1998) Firewalking: A Contemporary Ritual and Transformation. The Drama Review, 42, 98-114. https://doi.org/10.1162/dram.1998.42.2.98 https://www.google.it/url?sa=t\&rct=j\&q=\&esrc=s\&source=web\&cd=2\&cad=rja\&ua $\mathrm{ct}=8 \& v e d=2$ ahUKEwi80pDTvLHiAhVHW xoKHUMVBdoQFjABegQIARAC\&url= https\%3A\%2F\%2Flibres.uncg.edu\%2Fir\%2Funcg\%2Ff\%2FE_Edwards_Firewalking 1998.pdf\&usg=AOvVaw09-N6xXG7sZhxCX4Tor7Hs

[187] Jebbar, M., Franzetti, B., Girard, E. and Oger, P. (2015) Microbial Diversity and Adaptation to High Hydrostatic Pressure in Deep-Sea Hydrothermal Vents Prokaryotes. Extremophiles, 19, 721-740. https://doi.org/10.1007/s00792-015-0760-3 
https://www.researchgate.net/publication/279065389_Microbial_diversity_and_adapt ation_to_high_hydrostatic_pressure_in_deep-sea_hydrothermal_vents_prokaryotes

[188] Fisher, C.R., Takai, K. and Le Bris, N. (2007) Hydrothermal Vent Ecosystems. Oceanography, 20, 14-23. https://doi.org/10.5670/oceanog.2007.75

https://archimer.ifremer.fr/doc/2007/publication-6109.pdf 